\title{
Membrane transport in cell ensembles is modulated by the membrane state
}

Christoph Westerhausen ( $\nabla$ christoph.westerhausen@gmail.com )

University of Augsburg https://orcid.org/0000-0001-7103-7060

Nicolas Färber

University of Augsburg

Jonas Reitler

University of Augsburg

Julian Schäfer

University of Augsburg

\section{Biological Sciences - Article}

Keywords: Lipid membrane state, Order disorder phase transitions, Laurdan, Generalized polarization, Membrane permeability, Wound healing

Posted Date: February 3rd, 2022

DOI: https://doi.org/10.21203/rs.3.rs-1297077/v1

License: (c) (1) This work is licensed under a Creative Commons Attribution 4.0 International License. Read Full License 


\title{
Membrane transport in cell ensembles is modulated by the membrane state
}

\author{
Nicolas Färber ${ }^{1,2}$, Jonas Reitler², Julian Schäfer², Christoph Westerhausen 1,2,3, \\ 1 Experimental Physics I, Institute of Physics, University of Augsburg, Universitätsstraße 1, 86159 Augsburg, Germany \\ 2 Physiology, Institute of Theoretical Medicine, University of Augsburg, Universitätsstraße 2, 86159 Augsburg, Germany \\ 3 Center for NanoScience (CeNS), Ludwig-Maximilians-Universität Munich, 80799 Munich, Germany \\ corresponding author: christoph.westerhausen@gmail.com
}

Employing fluorescence microscopy, we measure the uptake of various fluorescent dyes into adherent HeLa cells and determine simultaneously the degree of membrane lipid chain order on a single cell level. The latter is measured by spectral analysis of the membrane-embedded dye Laurdan with different filter sets and quantified by determining the generalized polarization GP. First, we find that the mean GP value of single cells varies within a cell population in a range that is equivalent to a temperature variation of $9 \mathrm{~K}$. We exploit this natural variety of lipid membrane chain order to examine the uptake of fluorescent dyes as a function of GP at constant temperature. Using this approach, we experimentally show that transport across the cell membrane quantified by uptake of fluorescent dyes correlates with the membrane phase state. Specifically, we observed higher membrane transport with increasing lipid chain order. As a result, HeLa cells that adapted to lower culture temperatures by reducing lipid membrane chain order show less rhodamine $B$ transport than the $37^{\circ} \mathrm{C}$ culture. Aside from the cell intrinsic membrane phase state environmental factors have impact on the transport across the cell membrane as well. While temperature reduces lipid order, we found that locally high cell densities increase lipid order of cell membranes, and in turn leading to increased dye uptake. To demonstrate the physiological relevance of this concept, we analyzed cell phase state and transport at different stages of an in vitro wound healing process. While the uptake within a confluent cell layer is high due to the high degree of lipid chain order it decreases from the wound edge towards the wound center where the membrane lipid chain order is lowest.

\section{Keywords}

Lipid membrane state

Order disorder phase transitions

Laurdan

Generalized polarization

Membrane permeability

Wound healing 


\section{Introduction}

The lipid double layer of cell membranes is more than a mere barrier between cellular environments carrying proteins that account for all the membrane functions. The lipid molecules themself are an interacting system whose physical properties such as bending rigidity and viscosity depend on its phase state given by the lipid order [1]. Moreover, various examples indicate that lipids seem to foster particular cellular functions. For example the T-cell membrane lipid chain order increases during activation in the vicinity of activation sites and at the immunological synapse $[2,3]$. The membrane phase state influences adhesion as membrane lipid chain order is increased at focal adhesion points [4]. During virus budding highly ordered lipid regions are selectively incorporated into the influenza virus envelope [5]. Even virus infectivity depends on the lipid order of its envelope and can be reduced by cholesterol depletion [6] which is known to alter lipid order [7,8]. Cell membrane phase state dependent transport is so far mainly discussed in the context of lipid rafts that affect vesicular transport $[9,10]$. Lipid chain order transitions and temperature dependent cell membrane transport by passive diffusion however is pre-eminently object of cryobiology and sample cold storage [1114]. We want to focus on membrane transport as phase state dependent cellular function and were inspired by synthetically created lipid membrane model systems such as phospholipid vesicles and black lipid membranes which exhibit a permeability maximum at the phase transition between the gel and the fluid phase due to increased pore formation within the membrane at the phase transition $[15,16]$. Such transitions have been measured so far calorimetrically and optically in biological membranes as well $[14,17,18]$. For optical analysis one suitable membrane phase state probe is the fluorescent membrane-embedded dye Laurdan whose emission spectrum depends on the polarity and the relaxation dynamics of its environment $[19,20]$. The emission maximum shift of Laurdan from $440 \mathrm{~nm}$ to $490 \mathrm{~nm}$ upon decreasing lipid order can be quantified by the generalized polarization (GP) [21] which depends linearly on the lipid chain order [22]. Here, we determined GP on a single cell level by fluorescence microscopy and the use of two different emission filters to detect the wavelengths around $\lambda=440 \mathrm{~nm}$ and $\lambda=490 \mathrm{~nm}$ as shown in Fig. 1. For simultaneous determination of cell membrane transport we exposed adherent HeLa cells to rhodamine B and measured its fluorescence intensity after background reduction by rinsing in a third channel to quantify the dye uptake. State of the art image segmentation using the python module cellpose of Stringer et al. enabled us to ascribe pixel GP and pixel rhodamine $B$ intensity values to single cells and to calculate mean GP and mean rhodamine B intensities for each cell [23]. By comparison of the quantities GP and rhodamine $B$ uptake we answer the question whether membrane phase state and membrane transport are correlated. In a recent publication we analyzed the membrane phase state of Laurdan stained HeLa cell suspension over a wide temperature range and found a lipid chain order transition that extends over a temperature range from $-40{ }^{\circ} \mathrm{C}$ to $90{ }^{\circ} \mathrm{C}$ which is shown in Fig. $2 a$ [8]. If the permeability of cell membranes is highest at the phase transition as observed for synthetic lipid membranes, we expect increased rhodamine $B$ transport due to passive diffusion for cell membranes with a GP value of zero corresponding to an equal amount of ordered and disordered lipid chains, meaning the phase transition.

The structure of this study is as follows. First, we examine the cell membrane permeability by rhodamine $B$ uptake in various GP ranges by varying the temperature. Second, the rhodamine $B$ uptake of HeLa cells that adapted lipid chain order to hypothermal culture temperature and have in consequence a lower GP value is compared to cells 


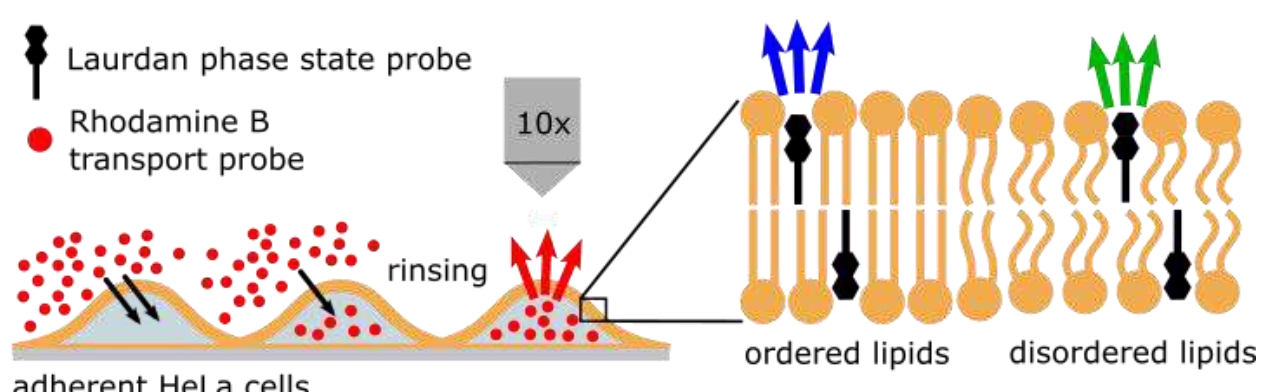

adherent HeLa cells
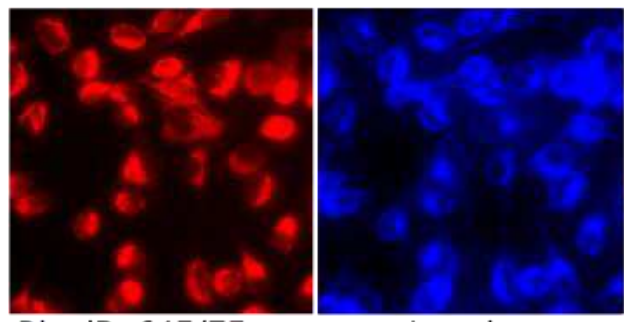

RhodB 645/75 nm

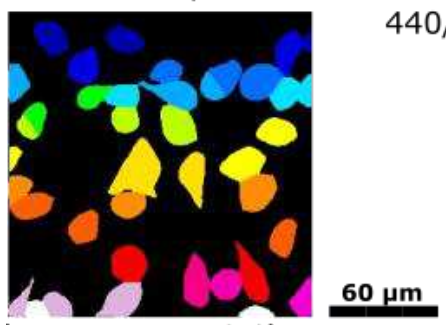

image segmentation

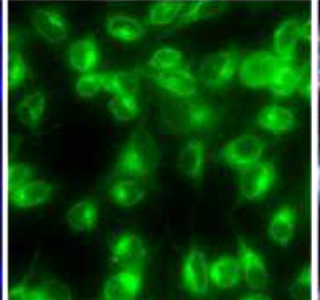

Laurdan

$490 / 40 \mathrm{~nm}$

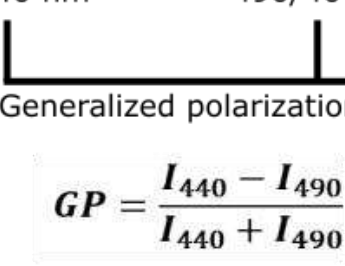

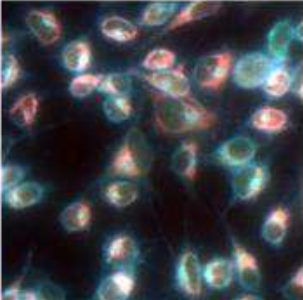

composite

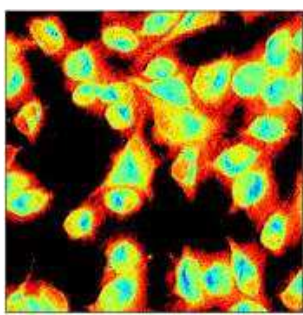

high GP

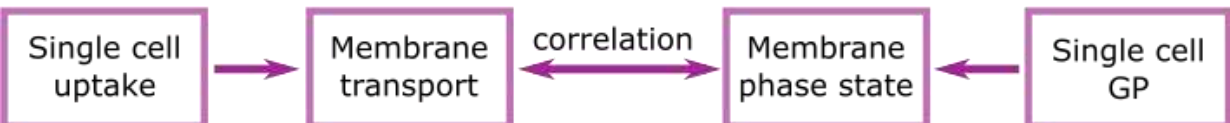

Figure 1 Experimental procedure and data analysis. The concept of simultaneous analysis of membrane phase state and membrane transport is visualized in the sketch above. The emission spectrum of the membrane-embedded dye Laurdan shifts from blue $(440 \mathrm{~nm})$ to greenish $(490 \mathrm{~nm})$ with increasing lipid disorder therefore indicating the membrane phase state while the fluorescence intensity of rhodamine B acts as a measure for the amount of cargo transported into the cell. The spectral changes of Laurdan are measured using of two different emission filters and quantified by the generalized polarization (GP). Image segmentation performed by a neural network allows for determination of single cell uptake and GP values. With these we answer the question whether cell membrane phase state and transport across the membrane are correlated.

cultured at standard conditions. Third, we validate the rhodamine $B$ results by time resolved transport measurements using Hoechst 33342 as membrane transport probe. Ultimately, the membrane phase state and transport dependency on local cell density is analyzed and its physiological relevance is demonstrated in the context of wound healing.

\section{Results and Discussion}

In this study we conducted membrane transport measurements of adherent HeLa cells within the physiological temperature regime from $10^{\circ} \mathrm{C}$ to $37^{\circ} \mathrm{C}$ which is located right in the middle of the lipid chain order transition of a HeLa cell suspension shown in Fig. 2a. In order to examine whether the transition regime of adherent cells differs from the one of suspended cells, we determined the phase state of adherent HeLa cells in a temperature gradient setup that is shown in Fig. $2 b$. The difference between phase transitions of biological and synthetic membranes can be clearly seen in the fluorescence emission colour of Laurdan pictured below the gradient setup. While the phospholipid vesicles display a steep transition from greenish to blue indicating a sudden change of lipid chain order with respect to temperature the transition of HeLa cell membranes is much broader and continuous. The comparison of the GP change per temperature of 
a
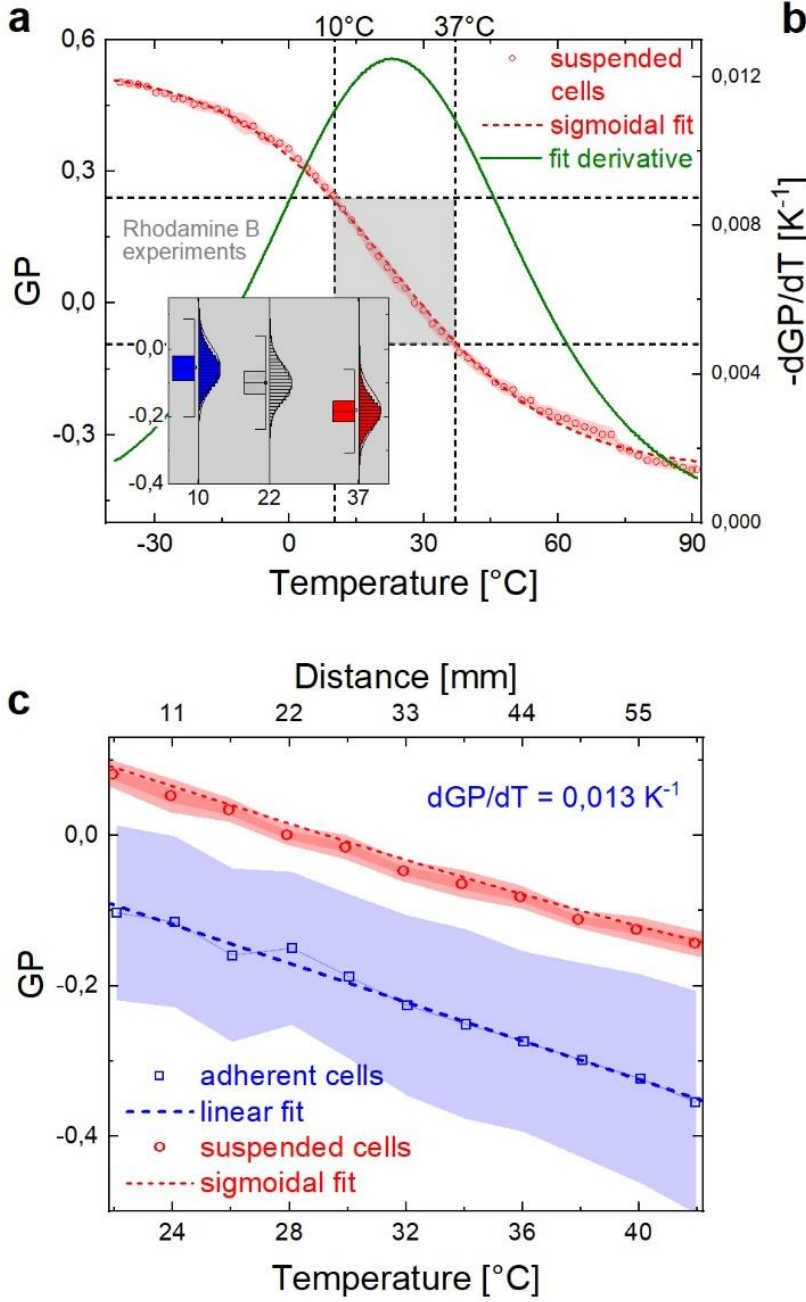

b

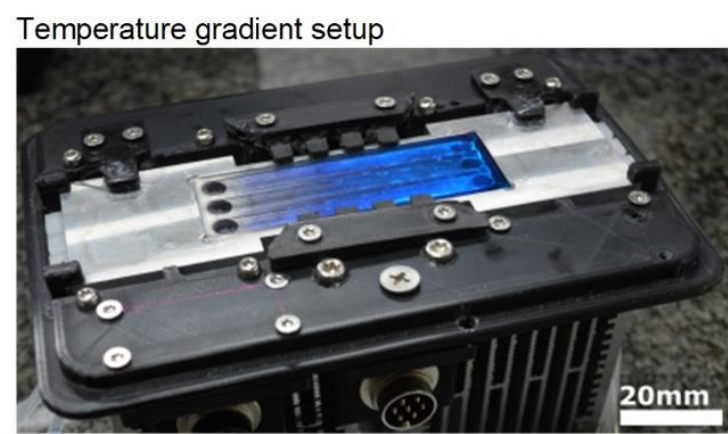

Laurdan stained vesicles

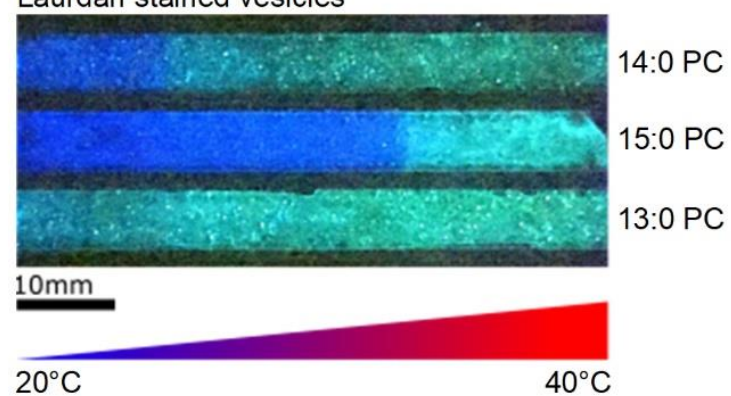

Laurdan stained cells

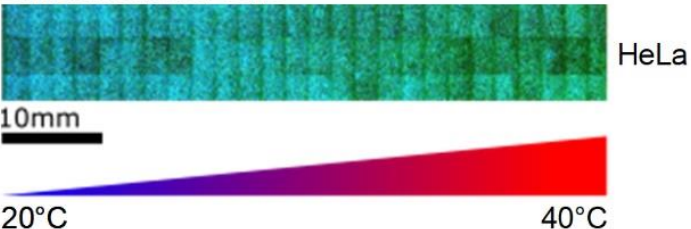

Figure 2 Temperature dependent phase state of adherent cells. a The temperature dependent generalized polarization of suspended HeLa cells and its derivative with respect to temperature show that HeLa cell membranes undergo a phase transition that extends from $-30^{\circ} \mathrm{C}$ to $90^{\circ} \mathrm{C}$. The permeability measurements conducted in this study were carried in a more physiological temperature regime $\left(10,22\right.$ and $\left.37^{\circ} \mathrm{C}\right)$ that is located right in the center of the broad phase transition where temperature changes have the biggest impact on lipid chain order. $\mathbf{b}$ Merged photography (room lighting and UV lamp) of a aluminum block that allows for temperature gradient exposure of cell overgrown glass slides via peltier cooling/heating on the backside. While vesicles consisting of pure phospholipids show sharp lipid order transitions indicated by the abrupt change of Laurdan emission colour HeLa cells undergo a far less steep transition. $\mathbf{c}$ The temperature dependent GP of the suspended cells shown in a) is plotted in a physiological temperature regime together with the GP of adherent cells. Both show a similar slope of $0.013 \mathrm{~K}^{-1}$ but a difference of absolute GP. In case of the suspended cells, symbols indicate the mean of three independent experiments in which one global phase state value per temperature point of a cell suspension was measured. The red bands show standard deviation and standard error. In case of the adherent cells, symbols represent the mean value of three independent experiments containing each 5000 cells per temperature interval. The blue coloured band indicates the standard deviation. The standard error band is too narrow to be shown here.

suspended and adherent cells shown Fig. $2 \mathrm{c}$ yields that except for the absolute GP value the steepness of the transition is in both cases similar with a slope of $\frac{\mathrm{dGP}}{\mathrm{dT}}=$ $0.013 \mathrm{~K}^{-1}$ which allows for conversion of GP changes to equivalent temperature changes. While the measurement of suspended cells yields only a mean value of the whole cell ensemble, the analysis of adherent cells by fluorescence microscopy allows for single cell uptake and phase state determination. By this we were able to measure the broadness of the GP distribution shown in Fig. $2 \mathrm{C}$ as the standard deviation of the single cell GP values coloured in blue, whereas the red coloured band indicates the standard deviation between the three independent measurements of suspended cells. This natural variance in membrane lipid chain order enabled us to measure rhodamine $B$ uptake as measure for membrane transport 
as function of membrane phase state quantified by GP.

We have chosen different dyes to examine the transport process into HeLa cells due to experimental constrictions when measuring GP and dye uptake intensity within one experiment. While the rhodamine emission spectrum does not interfere with the Laurdan emission allowing for simultaneous imaging of both dyes, it is still necessary to minimize background fluorescence by rinsing. Moreover, for the compensation of its temperature dependent quantum yield we recorded a calibration curve shown in SI-Fig. 1a Furthermore we analyzed whether rhodamine $B$ has any influence on the cell membrane phase state. Indeed, we observed that rhodamine $B$ exposure increases lipid order in average by 0.04 as shown in SI-Fig. 1b which is well below the effects that will be discussed in the following passage. But to eliminate the influence of the permeability probe on the membrane phase state we have conducted uptake experiments with Hoechst 33342 that only fluoresces after transport into the cells making it possible to record kinetics without rinsing steps. In this case the Laurdan stained membrane was imaged before Hoechst 33342 addition to avoid crosstalk.

\subsection{Temperature and phase state dependent rhodamine $B$ uptake}

The first observation of the single cell Laurdan phase state analysis is that the GP value averaged over one cell varies within a cell population at constant temperature in a GP range of about $\mathrm{FWHM}_{\mathrm{GP}}=0.12$ (Fig.3a). Considering the GP dependency on temperature of $0.013 \mathrm{~K}^{-1}$ (Fig.2b) this can be associated with a change in lipid chain order induced by a temperature variation of $\mathrm{FWHM}_{\mathrm{T}}=9 \mathrm{~K}$. Such GP variations were reported by Parasassi et al. for other cell types as well [24]. The natural variety of the cell membrane phase state between single cells enabled us to examine the uptake of fluorescent dyes as a function of GP without the need for manipulating the membranes in any way. The result of this analysis recorded at a temperature of $22^{\circ} \mathrm{C}$ is shown in Fig. 3c. We observed a monotonic increase of rhodamine $B$ uptake into the cells with increasing lipid chain order measured by GP. The maximum uptake is located at a GP value around zero but only few data points could be collected in this region as the GP distribution does not extend into the range above GP $=0$ (Fig. 3a) at $\mathrm{T}=22^{\circ} \mathrm{C}$. To verify that there is indeed a GP dependent rhodamine B uptake and not only a GP dependency on rhodamine $B$ we use a linear fit to the data of the $22^{\circ} \mathrm{C}$ experiment in Fig. $3 \mathrm{c}$ and calculate that rhodamine $\mathrm{B}$ would have to change GP by $2 \times 10^{-3}$ per count intensity. The comparison of the mean GP values of two Laurdan stained cell populations, one after rhodamine $\mathrm{B}$ exposure emitting a mean fluorescence of 122 counts and one reference, reveals a GP shift of 0.04 (SIFig. 1b) meaning that rhodamine $B$ shifts the cell membrane GP in average only by $3 \times 10^{-4}$ per count. Thus, the effect is two orders of magnitude lower. To exclude GP dependencies on cell size and in consequence on the rhodamine $B$ uptake we plotted GP as a function of the cell membrane area in SI-Fig. 2a. We found no pronounced size dependency and obtained a pearson-correlation coefficient of $r=0.03$. Furthermore, we analyzed the phase state dependent rhodamine $B$ uptake for different cell sizes and observed the same trend for every cell size as shown in SI-Fig. 2b.

A GP of zero implies that Laurdan emits just as much intensity at $440 \mathrm{~nm}$ as at $490 \mathrm{~nm}$ meaning that approximately an equal numbers of lipid chains are in the ordered and disordered state. In the context of lipid membrane phase state this indicates the phase transition regime. At this point synthetic lipid membranes exhibit the highest permeability as it was shown by Blicher et al. who measured the permeation rate of DPPC/DPPG vesicles as a function of temperature [16]. To examine the applicability of this concept to the far more complex system of cell membranes we studied the GP dependent rhodamine B uptake at lower temperatures where lipid 
a
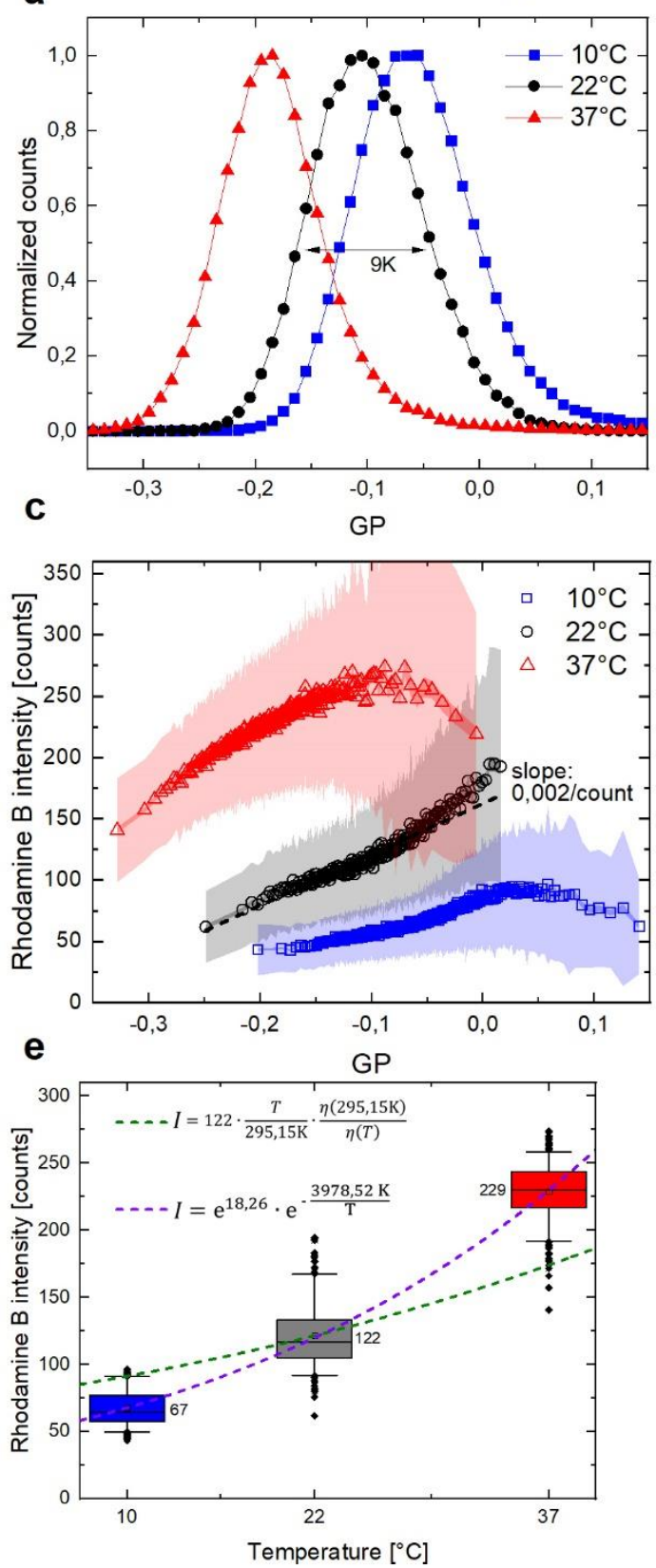

b

Phase state variation by adaption
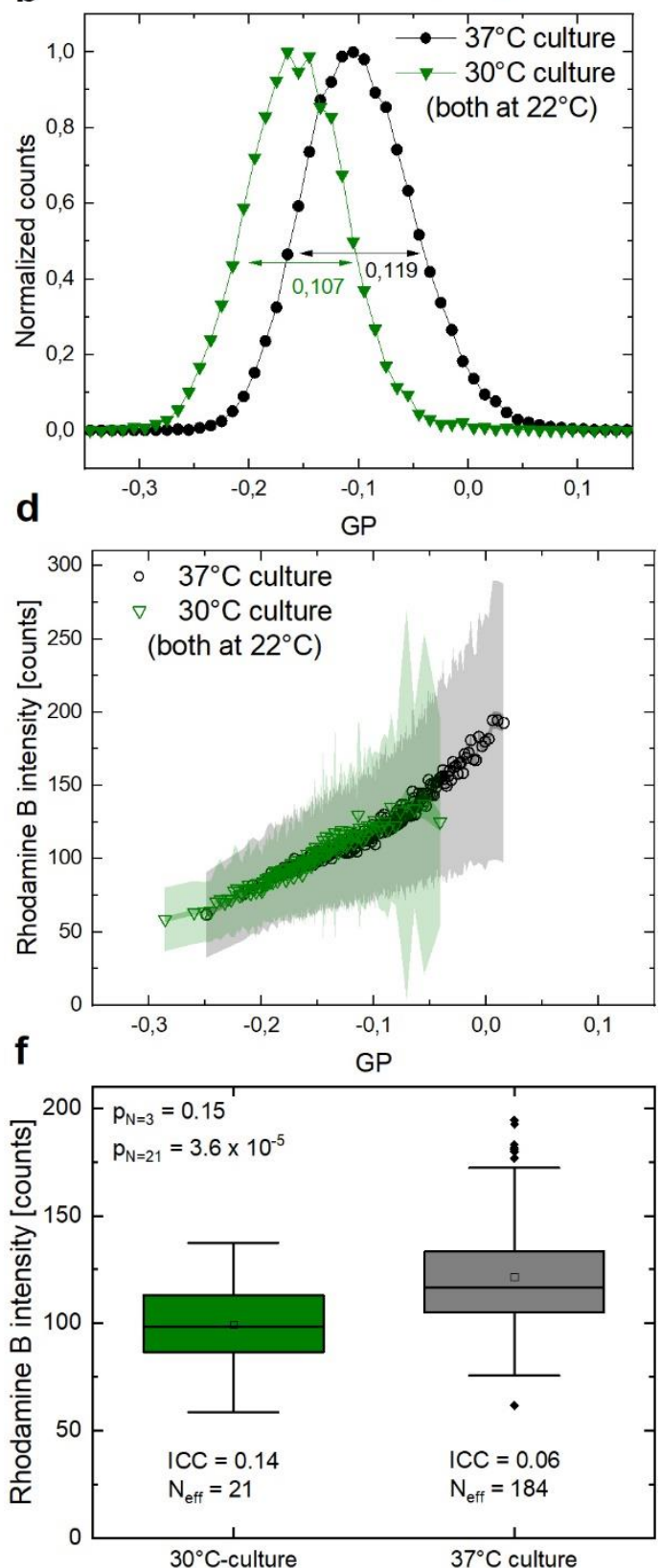

Figure 3 Phase state dependent rhodamine B uptake. a Distribution of single cell GP values within a population at three different temperatures. With increasing temperature the distributions shift towards lower GP values meaning less lipid chain order. Using the GP slope obtained in Fig. $2 \mathrm{~b}$ of $\mathrm{dGP} / \mathrm{dT}=0.013 \mathrm{~K}^{-1}$ the width of the histograms can be associated with a temperature interval of $9 \mathrm{~K}$ width. $\mathbf{b}$ Histograms of GP values of two differently cultured HeLa cell populations measured at the same temperature of $22^{\circ} \mathrm{C}$. The cells that were cultured at $30^{\circ} \mathrm{C}$ for one year exhibit less lipid chain order compensating the temperature induced increased lipid chain order during growth. $\mathbf{c}$ Rhodamine B intensity within cells as measure for cell membrane transport as function of GP measuring lipid chain order recorded at three different temperatures. High temperatures allow for investigation of low GP values meaning low lipid chain order and vice versa. The symbols represent the mean value of a GP interval of three independent experiments. The two colored bands indicate standard deviation and standard error. The temperature dependence of rhodamine B intensitiy was corrected $\mathbf{d}$ Comparison of rhodamine $B$ transport measurements as a function of GP of two differently cultured cell populations at the same temperature. Since the hypothermal adapted cells cover a lower GP range, it is possible to measure transport phenomena as function of phase state without temperature effects. e Box plot of the rhodamine B intensity plotted in c. The dotted line indicates the uptake behavior relative to $\mathrm{T}=22^{\circ} \mathrm{C}$ as a function of temperature and temperature dependent viscosity regarding only the diffusion coefficient of water. $\mathbf{f}$ Box plot of the rhodamine B intensity of cold adapted and reference HeLa cells. Since the adapted population covers a lower GP range in $d$ that is associated with lower dye uptake the mean rhodamine B intensity is lower than for the reference cells. The first $\mathrm{p}$-value was calculated using a sample size of $\mathrm{N}=3$ corresponding to three independently conducted experiments. For the second calculation we used the effective sample size of 21 obtained by ICC analysis. 
GP distribution extends to higher values. The result is shown in Fig. 3a. The increasing emission intensity of rhodamine $\mathrm{B}$ with decreasing temperature was corrected using the calibration curve in SI-Fig. 1a The GP histogram recorded at $10^{\circ} \mathrm{C}$ is clearly shifted towards higher values compared to the $22^{\circ} \mathrm{C}$ sample enabling us to study the membrane transport beyond a GP value of zero. Indeed, the uptake of rhodamine $B$ shows a maximum around $\mathrm{GP}=0.025$ and decreases again with increasing lipid chain order. Therefore, the results of the $\mathrm{T}=10^{\circ} \mathrm{C}$ and $\mathrm{T}=22^{\circ} \mathrm{C}$ measurements could be described by a model of phase state dependent passive diffusion through the cell membrane lipid bilayer.

To cover the most relevant phase state regime we conducted further experiments at a temperature of $\mathrm{T}=37^{\circ} \mathrm{C}$. Due to temperature induced reduced lipid chain order the GP distribution in Fig. 3a is shifted towards lower values. In line with the passive diffusion model an increasing dye uptake from a GP of $\mathrm{GP}=-0.3$ to $\mathrm{GP}=-0.1$ can be seen in Fig. 3c. This trend is followed by decreased uptake towards zero GP. Based on this outcome we conclude, that in addition to passive diffusion active processes must be involved in the transport mechanism of rhodamine $B$ across the HeLa cell membrane as well. High magnification fluorescence micrographs shown in SI-Fig. 1c support this assumption as they show on the one hand uniform rhodamine $B$ intensity distributed over the whole cytosol, but on the other hand vesicular structures exhibiting high rhodamine $b$ intensity. The latter could indicate endocytosis like uptake as discussed in the literature in the context of lipid rafts $[9,10]$. To estimate the portion of vesicular rhodamine $B$ uptake we compared the mean and median intensities within the cells as the median is less sensitive to few small regions of high intensity (SI-Fig. 1d). As the comparison yields an intensity difference of only about $2 \%$ we conclude that passive diffusion across membrane pores is the main mechanism causing the phase state dependent rhodamine B uptake shown here.

The absolute values of rhodamine $B$ intensity in Fig. 3a increase with higher temperature which implies that the uptake is not only temperature dependent due to membrane properties. This effect can be seen in the intensity box plot of Fig. 3e even clearer where the uptake of the whole cell population is analysed GP independently. We assume that the diffusion of rhodamine $B$ itself through the extra and intracellular environment depends on temperature as well. In general, the diffusion coefficient of fluids is directly proportional to temperature and inversely proportional to viscosity which is temperature dependent as well. The result of this dependency applied to the $22^{\circ} \mathrm{C}$ uptake intensity as reference value is shown in Fig. $3 e$ as dotted line. While it describes our observations of increasing dye uptake with increasing temperature qualitatively well it still does not match the absolute values perfectly. Therefore, a model of unhindered rhodamine $B$ diffusion through membrane pores seems to be unlikely. Assuming that an activation energy is necessary to overcome the cell membrane leads to an Arrhenius ansatz as often used for membrane permeability determination [25,26]. SI-Fig. 2c shows the Arrhenius plot of the mean rhodamine $B$ intensity of the whole cell populations measured at $\mathrm{T}=10^{\circ} \mathrm{C}, \mathrm{T}=22^{\circ} \mathrm{C}$ and $\mathrm{T}=37^{\circ} \mathrm{C}$. The parameters obtained by a linear fit were incorporated in the Arrhenius equation and plotted in Fig. 3e. As the permeability data is well approximated by the Arrhenius equation we calculated the activation energies for three different GP intervals again by linear fitting of the Arrhenius plots in SI-Fig. 2d and obtained values ranging from 15 to $20 \mathrm{~kJ} / \mathrm{mol}$ as shown in SI-Fig. 2 e.

In summary, we have shown that independent of the temperature the transport of rhodamine B correlates with the membrane phase state. More precisely, the uptake increases over a wide GP range with increasing lipid chain order. At $\mathrm{T}=10^{\circ} \mathrm{C}$ and $\mathrm{T}=37^{\circ} \mathrm{C}$ we observed in addition local maxima that could be ascribed to passive 
diffusion or active transport mechanisms. Temperature affects this process in a way that it increases diffusion on one hand, but decreases lipid chain order on the other hand. In turn, the latter inhibits transport to a certain degree.

\subsection{Phase state dependent rhodamine B uptake into hypothermal adapted cells}

Based on the results discussed above environmental changes that reduce lipid order should reduce rhodamine B uptake as well. In the case of increasing temperature this effect is overruled by the increased dye diffusion. Earlier, we have shown that HeLa cells cultured at lower temperatures $\left(30^{\circ} \mathrm{C}\right)$ adapt their membrane phase state by decreasing lipid chain order [8]. Similar temperature adaption effects were observed in E. coli membranes, paramecium multimicronucleatum and giant plasma membrane vesicles of zebra fish [17,18,27]. A comparison of hypothermal adapted cells with their $37^{\circ} \mathrm{C}$ cultured analogues enabled us to study two cell populations with different phase states at the same temperature. The effect of adaption can be seen in Fig. 3b where the GP histogram of the cells cultured at $30^{\circ} \mathrm{C}$ is shifted towards lower GP values meaning decreased lipid chain order. Furthermore, the distribution broadness decreases by $10 \%$ because of adaption which could indicate that the subpopulation of cells with high GP values is reduced stronger than the subpopulation with lower GP values in addition to a shift of the whole population. The rhodamine B uptake shown in Fig. 3d follows the same GP dependency as discussed for the $37^{\circ} \mathrm{C}$ culture. The rhodamine $B$ uptake increases towards higher GP values showing no local maximum as observed for cells examined at $\mathrm{T}=10^{\circ} \mathrm{C}$ or $\mathrm{T}=37^{\circ} \mathrm{C}$. The absolute rhodamine $B$ intensities match very well in the overlap region. Thus, the only difference between both samples is that in case of the adapted cells more cells are located at lower GP values corresponding to lower rhodamine $B$ uptake. This effect is reflected in the box plot of Fig. $3 f$ that shows the GP independent uptake of the whole cell populations. Because of the correlation of membrane phase state and membrane transport the cell population with lower lipid chain order shows less dye uptake.

\subsection{Phase state dependent Hoechst 33342 transport kinetics}

To study the phase state dependent membrane transport time resolved and to validate the rhodamine $B$ uptake experiments we studied the uptake of the widely used Hoechst 33342 nucleus staining dye. Hoechst 33342 was used as membrane transport probe by Lalande et al. before and facilitates the measurement procedure as no rinsing steps are necessary because it only fluoresces after intercalation into DNA [28]. As shown in Fig. $4 a$ the Laurdan stained cell membranes were imaged prior to Hoechst 33342 addition allowing for analysis of the uncompromised membrane and avoiding channel cross talk. The Laurdan imaging recorded at $\mathrm{T}=22^{\circ} \mathrm{C}$ yields as before a GP distribution that is shown in Fig. 4b. Directly after the phase state analysis Hoechst 33342 was added to the adherent cells and the increasing fluorescence intensity of the nucleus was imaged consequently every 6 minutes. The GP dependent uptake measured by the fluorescence intensity is plotted in Fig. 4b as well. The fluorescence increase is strongest at the beginning and saturates after 30 minutes. In addition, the uptake of cells with higher GP value is faster as compared to cells with low GP. To quantify this observation, we binned datapoints of equal GP and plotted their mean intensity as function of time shown in Fig. 4c. As a result of a linear approximation of the beginning intensity increase, we obtained different permeation rates for cells of different GP values. By this we can characterize membrane transport measured by the permeation rate as a function of phase state measured by GP as can be seen in Fig. 4d. With increasing GP meaning increasing lipid chain order the time constant measuring the permeation rate is increasing as well. Therefore, the 

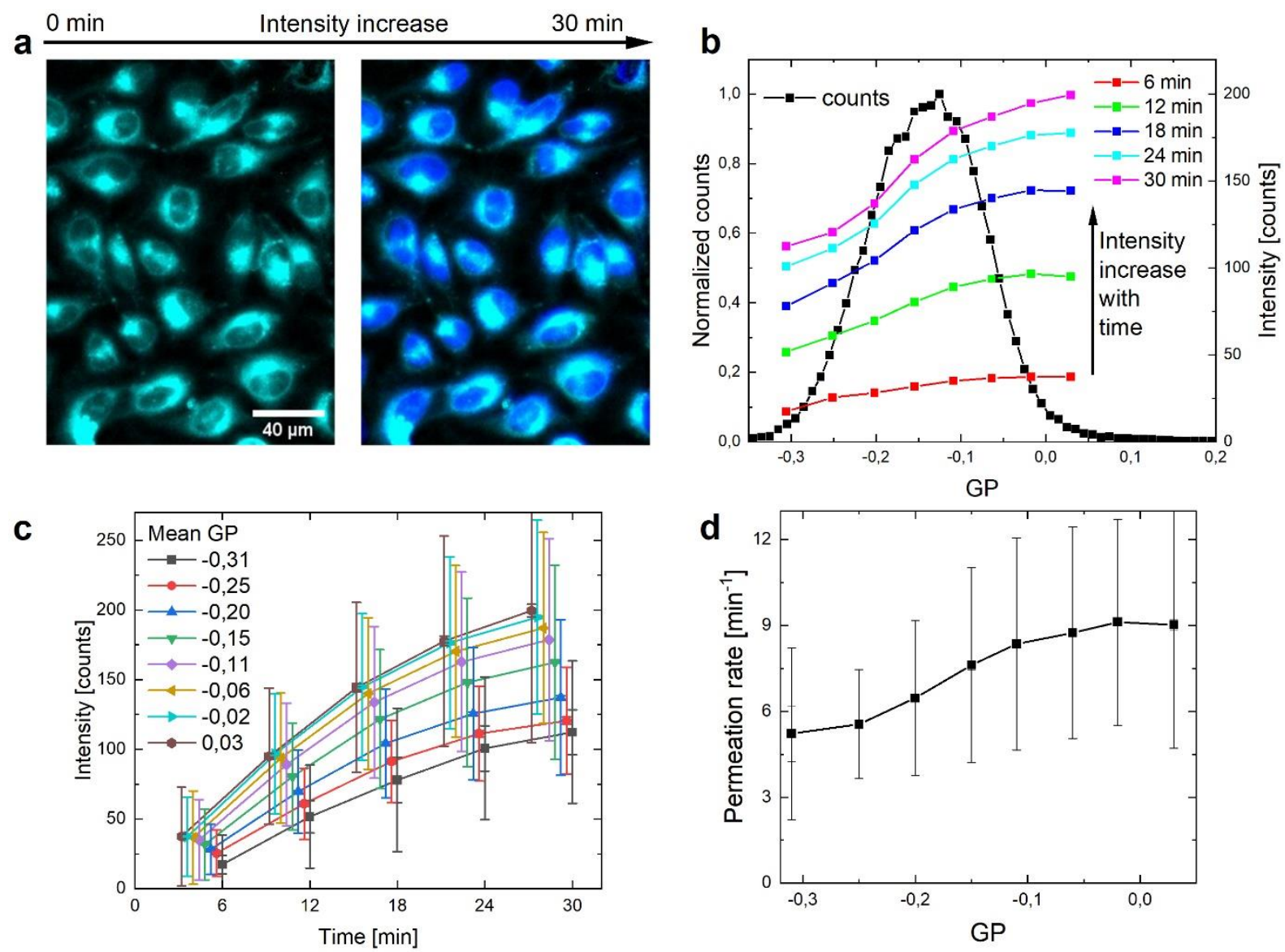

Figure 4 Phase state dependent Hoechst 33342 uptake. a Fluorescence micrograph of Laurdan (cyan) stained HeLa cell membranes prior and after addition of Hoechst 33342 (blue). b GP distribution as the normalized sum of three independent experiments and time course of GP dependent Hoechst 33342 mean intensity. c Hoechst 33342 mean intensity as function of time for different mean GP values. Error bars indicate standard deviation and standard error. Each graph is shifted by 0.04 minutes for better visibility. $\mathbf{d}$ Permeation rate as function of GP obtained by fitting of the data set presented in c.

Error bars indicate standard deviation and standard error.

correlation of membrane phase state and membrane transport holds not only for rhodamine $B$ but for Hoechst 33342 as well. As the DNA content as binding site of Hoechst 33342 may depend on the nucleus size we examined the GP dependence on the cell nucleus size in SI-Fig. 2f. We could not identify a pronounced correlation between these quantities and obtained a pearson-correlation coefficient of $r=0.01$.

\subsection{Cell density dependent membrane phase state and transport}

Based on the fact, that permeability and and lipid chain order are correlated, the question arises whether environmental changes that influence the cell membrane phase state would have impact on the membrane transport. To encounter this question, we first focused on a parameter that varied naturally within our samples: the local cell density. In our study to lipid phase transitions in cell membranes we found that morphological changes such as the transition from adherent to suspended during trypsinization were accompanied by changes in lipid order measured by GP [8]. Therefore, we expected cell deformation induced by spatial confinement of neighboured cells should affect GP as well. To analyze the local environment of single cells we defined cells with a distance of less than $31 \mu \mathrm{m}$ between their centers of mass as neighboured cells. Then, we determined for each cell the number of neighbours as measure for local cell density (Fig. 5b) and analyzed the membrane phase state by determining GP. The result for cells exclusively stained with Laurdan at $\mathrm{T}=22^{\circ} \mathrm{C}$ is shown in Fig. 5a. The mean GP value increases from $\mathrm{GP}=-0.17$ for cells without near neighbours up to $\mathrm{GP}=-0.03$ for cells 
a

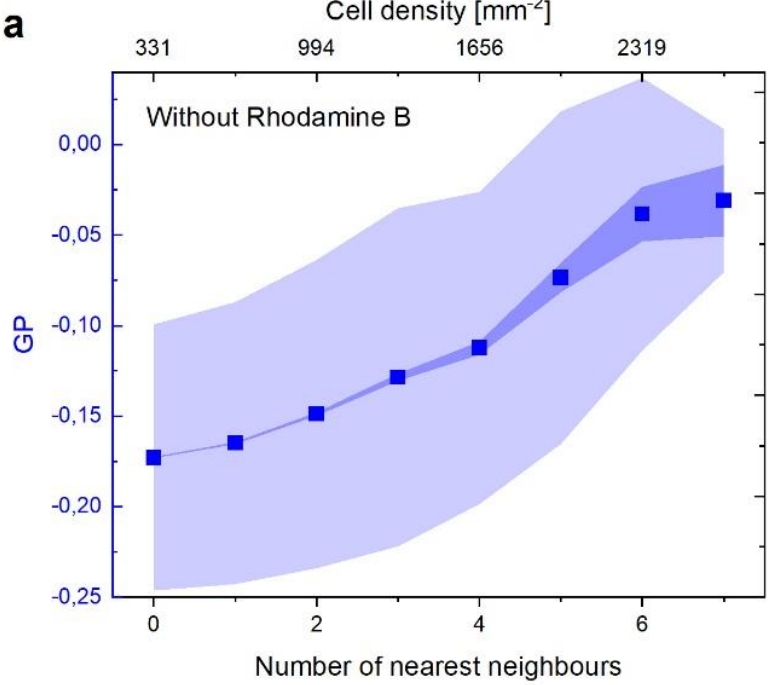

C

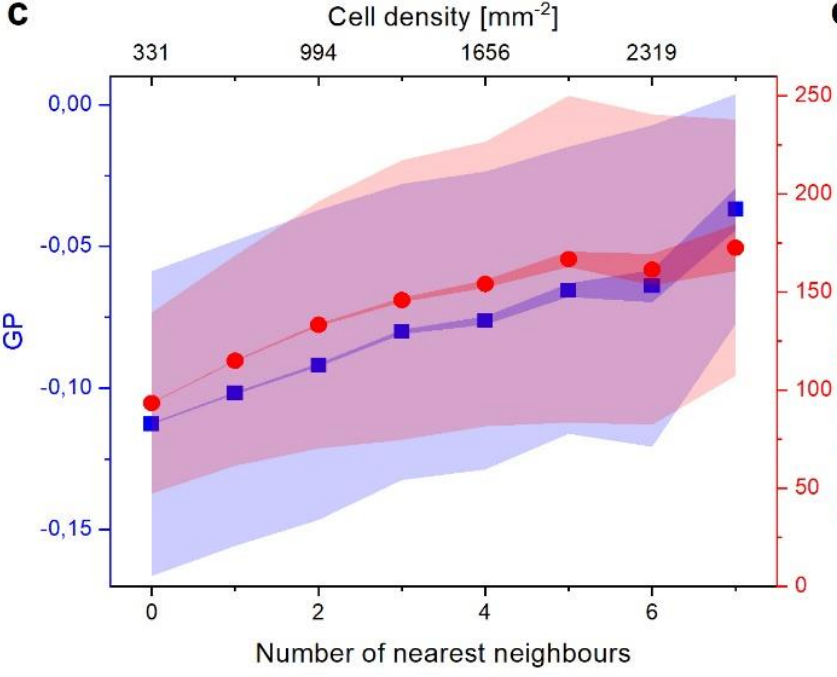

b

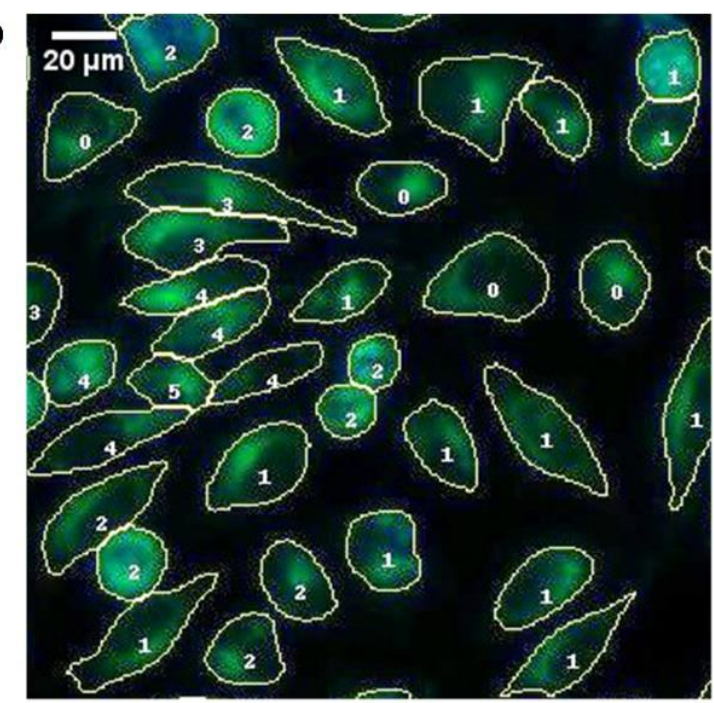

d

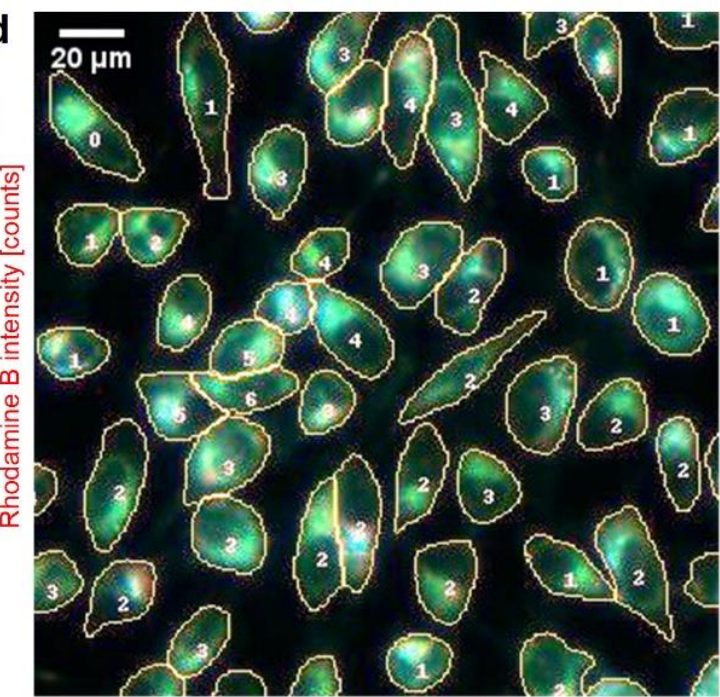

Figure 5 cell membrane phase state and transport dependency on cell density. a Mean cell GP as a function of the local cell density without rhodamine B determined by the number of nearest neighbours (center of mass within a radius of $31 \mu \mathrm{m}$ ). The coloured bands represent standard deviation and standard error. b Fluorescence microscopy 2-channel image of HeLa cells stained with Laurdan and segmentation outlines labelled with the number of nearest neighbours. c Mean cell GP and mean rhodamine $B$ intensity as a function of the number of cell neighbours. Lipid chain order increases with the number of neighbours causing the rhodamine B transport to increase as well. d Fluorescence microscopy 3-channel image of HeLa cells stained with Laurdan and rhodamine B.

with seven neighbours, meaning that spatially more confined cells exhibit more lipid order than cells without surrounding cells. This is in agreement with findings of Steinkühler et al. who measured increased lipid order in giant plasma membrane vesicles of cells cultured at different cell densities [1]. These changes in lipid order are likely to arise from alterations of the membrane lipid composition induced by the local cell environment [29,30].

To examine how cell density affects the membrane transport properties we executed the nearest neighbour analysis on Laurdan stained cells after rhodamine B exposure at $\mathrm{T}=22^{\circ} \mathrm{C}$ yielding not only neighbour dependent phase state but dye uptake as well. In Fig. $5 \mathrm{c}$ the phase state analysis reveals that the mean GP of single cells after rhodamine $B$ exposure increases again monotonically from $\mathrm{GP}=-0.11$ for zero neighbours up to $\mathrm{GP}=-0.04$ for cells surrounded by seven other cells. The slightly elevated GP values are probably a result of the phase state influence of rhodamine B itself. But, the remarkable finding is that, the membrane transport, measured by rhodamine B uptake, increases monotonically with increasing number of cell neighbours, too. This agrees with our results obtained by analysing the 

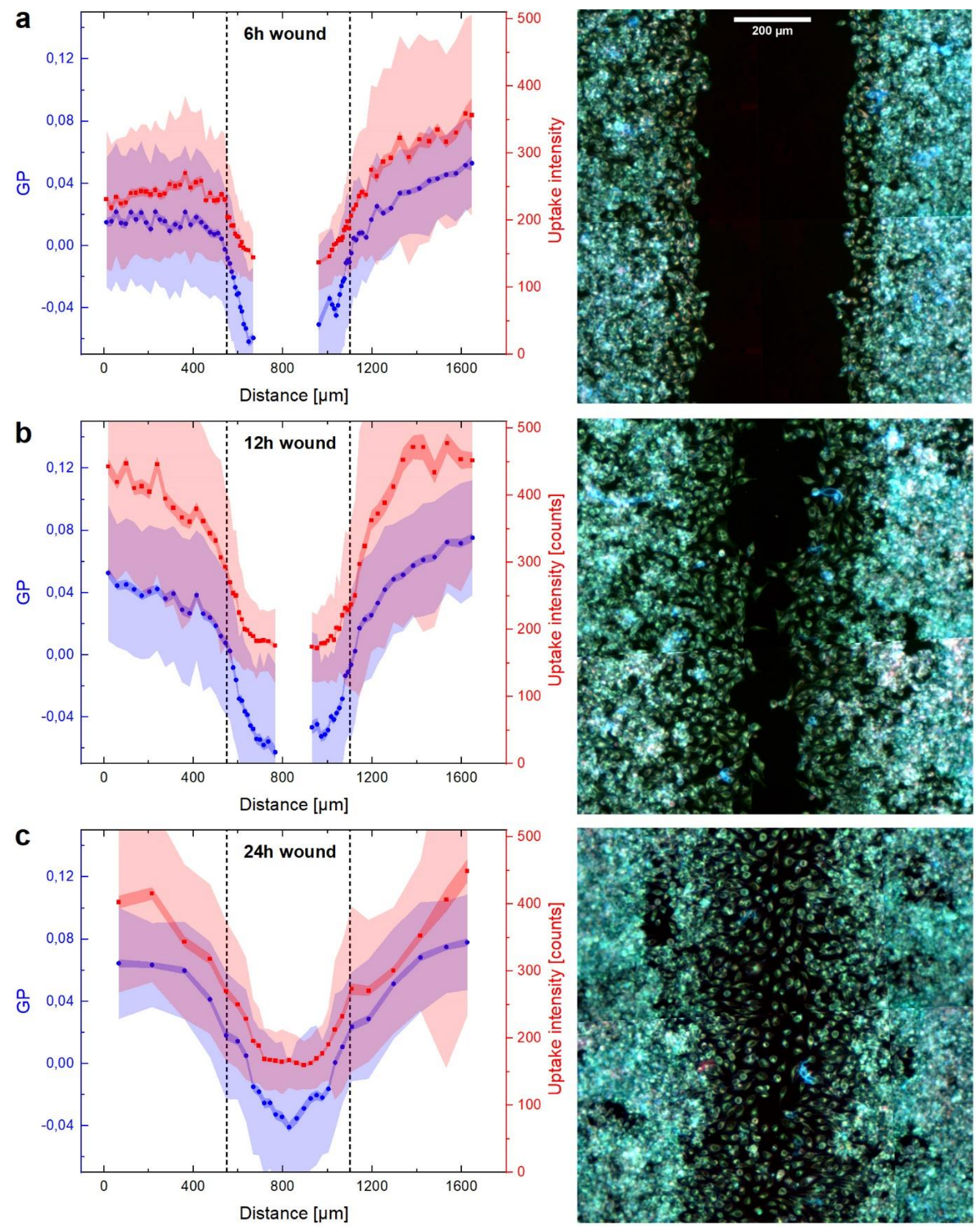

Figure 6 Phase state and transport analysis in the context of a wound healing process. a Mean GP and rhodamine B intensity as function of horizontal position within the wound healing assay pictured on the right side. The image was recorded and analyzed 6 hours after wound creation by removal of a silicon culture insert. The initial borders of the wound are indicated in the graph on the left as dotted vertical lines. The coloured bands indicate standard deviation and standard errors of the according quantities. As observed in the nearest neighbour analysis shown in fig. $5 \mathrm{c}$ GP and rhodamine $\mathrm{B}$ intensity decrease towards the wound center corresponding to lower cell density. $\mathbf{b}$ Wound phase state analysis 12 hours after culture insert removal. The wound closure is more advanced compared to the data of fig. 6a. c After 24 hours HeLa cells migrated into the wound center, but the cell density is still much lower compared to the confluent regions to the left and to the right. As a result, lipid chain order and rhodamine B uptake are still decreased in the wound center. 
rhodamine B and Hoechst 33342 at $\mathrm{T}=22^{\circ} \mathrm{C}$, where we observed the same GP dependent monotonic increase of dye uptake. Therefore, we conclude that due to the correlation of cell membrane phase state and membrane transport capabilities, environmental factors that influence the cell membrane phase state have a direct impact on cell membrane transport.

To demonstrate the physiological relevance of this concept, we applied it to a wound healing assay. Here, a steep cell density gradient is present between a confluent cell layer and a cell free gap, simulating an artificial wound. Experimentally this was achieved by removal of a silicone culture insert and imaging of the wound after staining with Laurdan and rhodamine $\mathrm{B}$. To monitor the process of healing we analyzed three different timepoints after removal of the culture insert: $t=6, t=12$ and $t=24$ hours. The GP value 6 hours after wound creation is displayed in Fig. $6 a$ as a function of horizontal position within the wound assay shown on the right side as a fluorescence micrograph. In the high cell density regions of the confluent cell layer left and right to the cell free area the mean GP reaches values of up to $\mathrm{GP}=0.04$ meaning high lipid chain order, while the mean GP within the artificial wound is as low as GP=-0.06 meaning low lipid chain order. As predicted from our findings on the correlation of GP and permeability above, the rhodamine $B$ uptake follows the GP distributions in these regions. During this time interval, the cells migrated about $100 \mu \mathrm{m}$ towards the cell free gap during wound healing. In this region around the initial wound border the rhodamine $B$ uptake decreases with decreasing cell density towards a minimum of 140 counts. Interestingly, cells with low plasma membrane bending rigidity corresponding to decreased lipid order, as reported by Steinkühler et al. [1], show increased migration [31]. Therefore, it is possible that the reduced lipid chain order of cells located at the wound border with a low number of neighbours contributes to the initiation of cell migration and thus the wound healing process. After 12 hours as shown in Fig. 6b the lipid order and membrane transport in the confluent region is even more increased as compared to the 6 hours measurement. This might be due to even higher cell density because of further cell proliferation. At this time point cells migrated about $200 \mu \mathrm{m}$ into the cell free gap. The decrease of lipid order and rhodamine $B$ uptake in the region from the initial wound border towards the wound center is less steep as compared to the 6 hours sample as the gradient from maximal cell density in the confluent region to zero density in the cell free gap extends over a larger distance of $200 \mu \mathrm{m}$. Even though the wound is closed after 24 hours which can be seen on the micrograph on the right side of Fig. $6 \mathrm{c}$ there is still a large difference in cell density in the image center as compared to the border. This is reflected in the spatially dependent GP and rhodamine $B$ uptake in Fig. $6 \mathrm{c}$ on the left side as well where the lipid order and dye uptake are still minimal in the wound center. But compared to the 6 and 12 hours measurements the minimum GP values are slightly enhanced as cell density starts to increase even in the wound center.

In the context of wound healing, we have shown that high cell densities induce ordering of the lipid chains which leads again to an increase of transport across the membrane. As a maybe counterintuitive result cells that do not have any neighbours and which are therefore much more exposed to any environmental influences show much less dye uptake than cells within a dense cell layer.

\section{Conclusion}

In summary we have shown that there is a direct proportionality of cell membrane lipid chain order to the transport across the cell membrane in a wide range of GP. As consequence, cells in a single cell ensemble with less lipid order of their membrane are less likely to take up cargo from the environment compared to cells with a higher lipid chain order at the same temperature. Moreover, we demonstrated 
that environmental factors that influence the physical cell membrane state such as cell density directly affect membrane transport. This finding allows for prediction of cell membrane transport properties under other environmental influences if their effect on the lipid phase state is known. These influences changing the lipid order of biological membranes of various cell types, for example, can be changes in $\mathrm{pH}$, solvent exposure, anesthesia and cholesterol content modulations as we have shown earlier [8]. Others have shown that lipid chain order measured by GP is altered by salt concentration or shear flow exposure [32,33]. In the context of nerve pulse propagation dynamic transitions from an unordered to an ordered lipid membrane state are discussed as signal carrier [34]. Our findings would further support this idea as it explains the increased ion permeability during the propagation of an action potential [35-37]. It would be very exciting to further elucidate the correlation of the cell membrane state and other cellular functions, as, e.g., enzyme activity, migration, adhesion or reversible deformation.

\section{Materials and Methods}

Fluorescence microscopy was performed on a Zeiss Axiovert 200M epi-fluorescence microscope. The Laurdan (Sigma-Aldrich Chemie GmbH, Munich, Germany) fluorescence was analyzed with different filter sets using the same excitation wavelength of $350 / 50 \mathrm{~nm}$ but different emission filters: $440 / 20 \mathrm{~nm}$ and $490 / 20 \mathrm{~nm}$. The latter was used for Hoechst 33342 (NucBlue $^{\mathrm{TM}}$, Sigma-Aldrich Chemie $\mathrm{GmbH}$, Munich, Germany) measurements as well. For imaging of rhodamine B (Sigma-Aldrich Chemie $\mathrm{GmbH}$, Munich, Germany) we utilized an excitation range of $585 / 30 \mathrm{~nm}$ and an $624 / 40 \mathrm{~nm}$ emission bandpass. The emission spectra of Laurdan, Hoechst 33342 and Rhodamine B were recorded using an Ocean optics QEPro spectrometer.

Image analysis started with stitching of single images using the Fiji plugin of
Preibisch et al. [38]. This was followed by image segmentation using the python package cellpose of stringer et al. [23]. The image intensity data was further processed in a python script. Cell mean GP was calculated by summing up the GP values of each single cell pixel using the according pixels in the $440 \mathrm{~nm}$ and $490 \mathrm{~nm}$ emission images followed by division by the number of cell pixels. The dye uptake intensity was determined in the same way as sum of pixel intensities normalized to the number of pixels. For the nearest neighbour analysis, the centroids of each cell were calculated as the center of mass. Surrounding cells were identified as neighbours if the Euclidean distance between the centers of mass was lower than $31 \mu \mathrm{m}$.

Hela cells (ATCC $\AA$ CCL-2 ${ }^{\text {TM }}$ ) were cultured in $25 \mathrm{~cm}^{2}$ NuncTM cell culture flasks (ThermoFisher Scientific, MA, USA) at $37^{\circ} \mathrm{C}$ in saturated atmosphere in DMEM (Bio\&SELL GmbH, Nürnberg, Germany) supplemented with $10 \%$ fetal bovine serum (FBS Superior) and $1 \%$ Pen/Strep (Biochrom $\mathrm{GmbH}$, Berlin, Germany). For fluorescence microscopy cells were trypsinated with $1 \mathrm{ml}$ of $0,05 \%$ trypsin/EDTA (Biochrom $\mathrm{GmbH}$, Berlin, Germany) and about 0.2 Mio. cells were transferred into a custom-made culture vessel with $5.5 \mathrm{~cm}^{2}$ glass bottom and incubated for 24 hours in culture medium. Prior to the uptake experiments cell membranes were stained in culture medium after addition of Laurdan dissolved in DMSO $(1 \mathrm{mg} / \mathrm{ml})$ for $2 \mathrm{~h}$ at 37 ${ }^{\circ} \mathrm{C}$. The final concentration of Laurdan and DMSO in the staining solution were $28 \mu \mathrm{M}$ and $141 \mathrm{mM}(11 \mathrm{mg} / \mathrm{l})$.

For Rhodamine B uptake measurements, the temperature of the Laurdan stained cells was adjusted to either $\mathrm{T}=10^{\circ} \mathrm{C}$, $\mathrm{T}=22^{\circ} \mathrm{C}$ or $\mathrm{T}=37^{\circ} \mathrm{C}$ with a custom-made microscope insert. After equilibration monitored with a pt100 temperature sensor tempered culture medium containing rhodamine $\mathrm{B}$ was added to the culture vessel yielding a dye concentration of $1 \mathrm{mM}$. After 10 minutes of rhodamine $B$ exposure the cells were thoroughly rinsed with tempered PBS buffer for at least 5 times. 3- 
channel imaging (Laurdan at 440nm, Laurdan at 490nm and rhodamine at $624 \mathrm{~nm}$ ) was carried out under PBS buffer at the according temperature.

Hoechst 33342 uptake measurements were carried out at $\mathrm{T}=22^{\circ} \mathrm{C}$. After Laurdan staining cells were directly imaged with two channels (Laurdan 440nm and Laurdan 490nm). Afterwards tempered culture medium containing Hoechst 33342 was added to the culture vessel yielding a dye concentration of $8 \mu \mathrm{M}$. Immediately after addition the increasing Hoechst 33342 fluorescence was recorded by consecutively 1-channel (Laurdan and Hoechst 33342 at $490 \mathrm{~nm}$ ) scans. During intensity data processing the Hoechst 33342 intensity was corrected for Laurdan intensity.

For Wound healing analysis we placed 2well silicone culture inserts (Ibidi $\mathrm{GmbH}$, Gräfelfing, Germany) with a growth area of $0.22 \mathrm{~cm}^{2}$ per well and a cell free gap of around $500 \mu \mathrm{m}$ onto glass slides. After filling each well with about 50,000 cells the slides were incubated for 24 hours followed by removal of the silicone insert. Laurdan staining was carried out after $t=6, t=12$ and $\mathrm{t}=24$ hours at $\mathrm{T}=37^{\circ} \mathrm{C}$ as described above. Afterwards the cells were exposed to $1 \mathrm{mM}$ Rhodamine $\mathrm{B}$ in culture medium for $10 \mathrm{~min}$ at $22^{\circ} \mathrm{C}$ followed by rinsing with PBS buffer for at least 5 times. Finally, three channels were imaged (Laurdan at 440nm, Laurdan at $490 \mathrm{~nm}$ and rhodamine at $624 \mathrm{~nm}$ ).

\section{Data representation}

All data shown originates from at least three independently conducted experiments. In the case of GP-histograms we used throughout a bin size of 0.01 and summed up the counts of three independent datasets each contributing the same number of cells as can be seen in the raw data plot in SI-Fig. 3. For comparison these histograms were normalized to zero and one as shown in Fig. 3,4. The according intensity datasets were reduced by sorting the GP and rhodamine B intensity tupels of all three experiments ascending according to GP and calculating the mean, standard deviation and standard error for a constant number of consecutive values. For this number we chose the square root of the number of all data points.

\section{Statistics}

Two tailed t-tests for $5 \%$ statistical significance between two values were performed with four degrees of freedom corresponding to six independently measured samples. In addition we calculated t-values with a higher degree of freedom using an effective sample size obtained by ICC analysis based on Liljequist et al. [39].

\section{Acknowledgements}

The authors thank Prof. Dr. Matthias Schneider, Simon Fabiunke and Achim Wixforth for fruitful discussions. The authors thank the Center for Nanoscience (CeNS) and the Augsburg Centre for Innovative Technologies (ACIT) for funding. C.W. would like to acknowledge funding for the project "Physical and functional interaction mechanisms at cell membranes and vessel walls" by the University of Augsburg. N.F. would like to thank the Joachim Herz foundation.

\section{Data availability}

All data that support our findings are available from the corresponding authors upon reasonable request. There are no restrictions on data availability.

\section{Author contributions}

N.F. and C.W. designed research; N.F., J.S., J.R. and C.W. performed research; N.F., J.S. and J.R. analyzed data and implemented experimental tools; N.F. and C.W. wrote the paper.

\section{Competing interests}

The authors declare no competing interests. 


\section{References}

[1] Steinkühler J, Sezgin E, Urbančič I, Eggeling C, Dimova R. Mechanical properties of plasma membrane vesicles correlate with lipid order, viscosity and cell density. Commun Biol 2019;2:337.

https://doi.org/10.1038/s42003-0190583-3.

[2] Gaus K, Chklovskaia E, Fazekas De St. Groth B, Jessup W, Harder $\mathrm{T}$. Condensation of the plasma membrane at the site of $\mathrm{T}$ lymphocyte activation. J Cell Biol 2005;171:121-31. https://doi.org/10.1083/jcb.2005050 47.

[3] Owen DM, Oddos S, Kumar S Davis DM, Neil MAA, French PMW, et al. High plasma membrane lipid order imaged at the immunological synapse periphery in live T cells. Mol Membr Biol 2010;27:178-89. https://doi.org/10.3109/09687688.20 10.495353.

[4] Gaus K, Le Lay S,

Balasubramanian N, Schwartz MA. Integrin-mediated adhesion regulates membrane order. J Cell Biol 2006;174:725-34. https://doi.org/10.1083/jcb.2006030 34.

[5] Scheiffele P, Rietveld A, Wilk T, Simons K. Influenza viruses select ordered lipid domains during budding from the plasma membrane. J Biol Chem 1999;274:2038-44. https://doi.org/10.1074/jbc.274.4.20 38.

[6] M. GDR, Elena C, M. HJ, O. AL, K. HJE. Cholesterol Depletion of Human Immunodeficiency Virus Type 1 and Simian Immunodeficiency Virus with $\beta$ Cyclodextrin Inactivates and Permeabilizes the Virions: Evidence for Virion-Associated Lipid Rafts. J Virol 2003;77:8237-48. https://doi.org/10.1128/JVI.77.15.82
37-8248.2003.

[7] laea DB, Maxfield FR. Membrane order in the plasma membrane and endocytic recycling compartment. PLoS One 2017;12:6-13. https://doi.org/10.1371/journal.pone. 0188041.

[8] Färber N, Westerhausen C. Broad lipid phase transitions in mammalian cell membranes measured by Laurdan fluorescence spectroscopy. Biochim Biophys Acta - Biomembr 2022;1864:183794.

https://doi.org/https://doi.org/10.101 6/j.bbamem.2021.183794.

[9] Hanzal-Bayer MF, Hancock JF. Lipid rafts and membrane traffic. FEBS Lett 2007;581:2098-104. https://doi.org/https://doi.org/10.101 6/j.febslet.2007.03.019.

[10] Ikonen E. Roles of lipid rafts in membrane transport. Curr Opin Cell Biol 2001;13:470-7.

https://doi.org/https://doi.org/10.101 6/S0955-0674(00)00238-6.

[11] Wolkers WF, Oldenhof H, Tang F, Han J, Bigalk J, Sieme H. Factors Affecting the Membrane Permeability Barrier Function of Cells during Preservation Technologies. Langmuir 2019;35:7520-8.

https://doi.org/10.1021/acs.langmuir .8b02852.

[12] Akhoondi M, Oldenhof $H$, Stoll $C$, Sieme H, Wolkers WF. Membrane hydraulic permeability changes during cooling of mammalian cells. Biochim Biophys Acta (BBA)Biomembranes 2011;1808:642-8.

[13] Wolkers WF, Crowe LM, Tsvetkova NM, Tablin F, Crowe JH. In situ assessment of erythrocyte membrane properties during cold storage. Mol Membr Biol 2002;19:59-65.

[14] Crowe JH, Hoekstra FA, Crowe LM, Anchordoguy TJ, Drobnis E. Lipid phase transitions measured in intact cells with fourier transform infrared 
spectroscopy. Cryobiology

1989;26:76-84.

https://doi.org/10.1016/0011 -

2240(89)90035-7.

[15] Cruzeiro-Hansson L, Mouritsen OG. Passive ion permeability of lipid membranes modelled via lipiddomain interfacial area. Biochim Biophys Acta - Biomembr 1988;944:63-72.

https://doi.org/https://doi.org/10.101 6/0005-2736(88)90316-1.

[16] Blicher A, Wodzinska K, Fidorra M, Winterhalter M, Heimburg T. The temperature dependence of lipid membrane permeability, its quantized nature, and the influence of anesthetics. Biophys $\mathrm{J}$ 2009;96:4581-91. https://doi.org/10.1016/j.bpj.2009.01 .062 .

[17] Toyoda T, Hiramatsu Y, Sasaki T, Nakaoka Y. Thermo-sensitive response based on the membrane fluidity adaptation in Paramecium multimicronucleatum. J Exp Biol 2009;212:2767-72.

https://doi.org/10.1242/jeb.031278.

[18] Mužić T, Tounsi F, Madsen SB, Pollakowski D, Konrad M, Heimburg $\mathrm{T}$. Melting transitions in biomembranes. Biochim Biophys Acta - Biomembr 2019;1861:183026. https://doi.org/10.1016/j.bbamem.20 19.07.014.

[19] Parasassi T, Conti F, Gratton E. Time-resolved fluorescence emission spectra of Laurdan in phospholipid vesicles by multifrequency phase and modulation fluorometry. Cell Mol Biol 1986;32:103-8.

[20] Parasassi T, Gratton E. Packing of phospholipid vesicles studied by oxygen quenching of Laurdan fluorescence. J Fluoresc 1992;2:167-74. https://doi.org/10.1007/BF00866931

[21] Parasassi, T and De Stasio, $G$ and Ravagnan, $G$ and Rusch, RM and
Gratton E. Quantitation of lipid phases in phospholipid vesicles by the generalized polarization of Laurdan fluorescence. Biophys $\mathrm{J}$ 1991;60:179-89.

[22] Leung SSW, Brewer J, Bagatolli LA, Thewalt JL. Measuring molecular order for lipid membrane phase studies: Linear relationship between Laurdan generalized polarization and deuterium NMR order parameter. Biochim Biophys Acta Biomembr 2019;1861:183053. https://doi.org/10.1016/j.bbamem.20 19.183053.

[23] Stringer $C$, Wang $T$, Michaelos $M$, Pachitariu M. Cellpose: a generalist algorithm for cellular segmentation. Nat Methods 2021;18:100-6. https://doi.org/10.1038/s41592-02001018-X.

[24] Parasassi T, Gratton E, Yu WM, Wilson P, Levi M. Two-photon fluorescence microscopy of laurdan generalized polarization domains in model and natural membranes. Biophys J 1997;72:2413-29. https://doi.org/10.1016/S00063495(97)78887-8.

[25] Weng L, Ellett F, Edd J, Wong KHK, Uygun K, Irimia D, et al. A highlyoccupied, single-cell trapping microarray for determination of cell membrane permeability. Lab Chip 2017;17:4077-88. https://doi.org/10.1039/c7lc00883j.

[26] Fleury JB. Enhanced water permeability across a physiological droplet interface bilayer doped with fullerenes. RSC Adv 2020;10:19686-92. https://doi.org/10.1039/d0ra01413c.

[27] Burns M, Wisser K, Wu J, Levental I, Veatch SL. Miscibility Transition Temperature Scales with Growth Temperature in a Zebrafish Cell Line. Biophys J 2017;113:1212-22. https://doi.org/10.1016/j.bpj.2017.04 .052 .

[28] Lalande ME, Ling V, Miller RG. Hoechst 33342 dye uptake as a probe of membrane permeability 
changes in mammalian cells. Proc Natl Acad Sci 1981;78:363 LP _ 367. https://doi.org/10.1073/pnas.78.1.36 3.

[29] Snijder B, Sacher R, Rämö P, Damm E-M, Liberali P, Pelkmans L. Population context determines cellto-cell variability in endocytosis and virus infection. Nature 2009;461:520-3.

https://doi.org/10.1038/nature08282

[30] Frechin M, Stoeger T, Daetwyler S, Gehin C, Battich N, Damm E-M, et al. Cell-intrinsic adaptation of lipid composition to local crowding drives social behaviour. Nature 2015;523:88-91. https://doi.org/10.1038/nature14429

[31] Braig S, Sebastian Schmidt BU, Stoiber K, Händel C, Möhn T, Werz $\mathrm{O}$, et al. Pharmacological targeting of membrane rigidity: implications on cancer cell migration and invasion. New J Phys 2015;17:83007.

https://doi.org/10.1088/13672630/17/8/083007.

[32] Vest RS, Gonzales LJ, Permann SA, Spencer E, Hansen LD, Judd AM, et al. Divalent Cations Increase Lipid Order in Erythrocytes and Susceptibility to Secretory Phospholipase A2. Biophys J 2004;86:2251-60. https://doi.org/https://doi.org/10.101 6/S0006-3495(04)74283-6.

[33] Yamamoto K, Ando J. Endothelial cell and model membranes respond to shear stress by rapidly decreasing the order of their lipid phases. J Cell Sci 2013;126:122734.

https://doi.org/10.1242/jcs.119628.

[34] Heimburg T, Jackson AD. The thermodynamics of general anesthesia. Biophys $\mathrm{J}$ 2007;92:3159-65.

https://doi.org/10.1529/biophysj.106 .099754 .
[35] Heimburg T. The capacitance and electromechanical coupling of lipid membranes close to transitions: The effect of electrostriction. Biophys J 2012;103:918-29. https://doi.org/10.1016/j.bpj.2012.07 .010 .

[36] Heimburg T, Jackson AD. On soliton propagation in biomembranes and nerves. Proc Natl Acad Sci U S A 2005;102:9790-5. https://doi.org/10.1073/pnas.050382 3102.

[37] Fillafer C, Paeger A, Schneider MF. The living state: How cellular excitability is controlled by the thermodynamic state of the membrane. Prog Biophys Mol Biol 2021;162:57-68. https://doi.org/https://doi.org/10.101 6/j.pbiomolbio.2020.10.003.

[38] Preibisch S, Saalfeld S, Tomancak P. Globally optimal stitching of tiled 3D microscopic image acquisitions. Bioinformatics 2009;25:1463-5. https://doi.org/10.1093/bioinformatic s/btp184.

[39] Liljequist D, Elfving B, Skavberg Roaldsen K. Intraclass correlationA discussion and demonstration of basic features. PLoS One 2019;14:e0219854. 
a

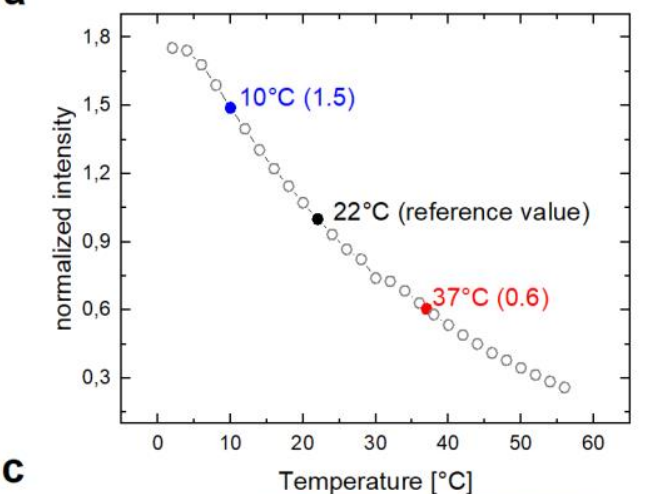

b
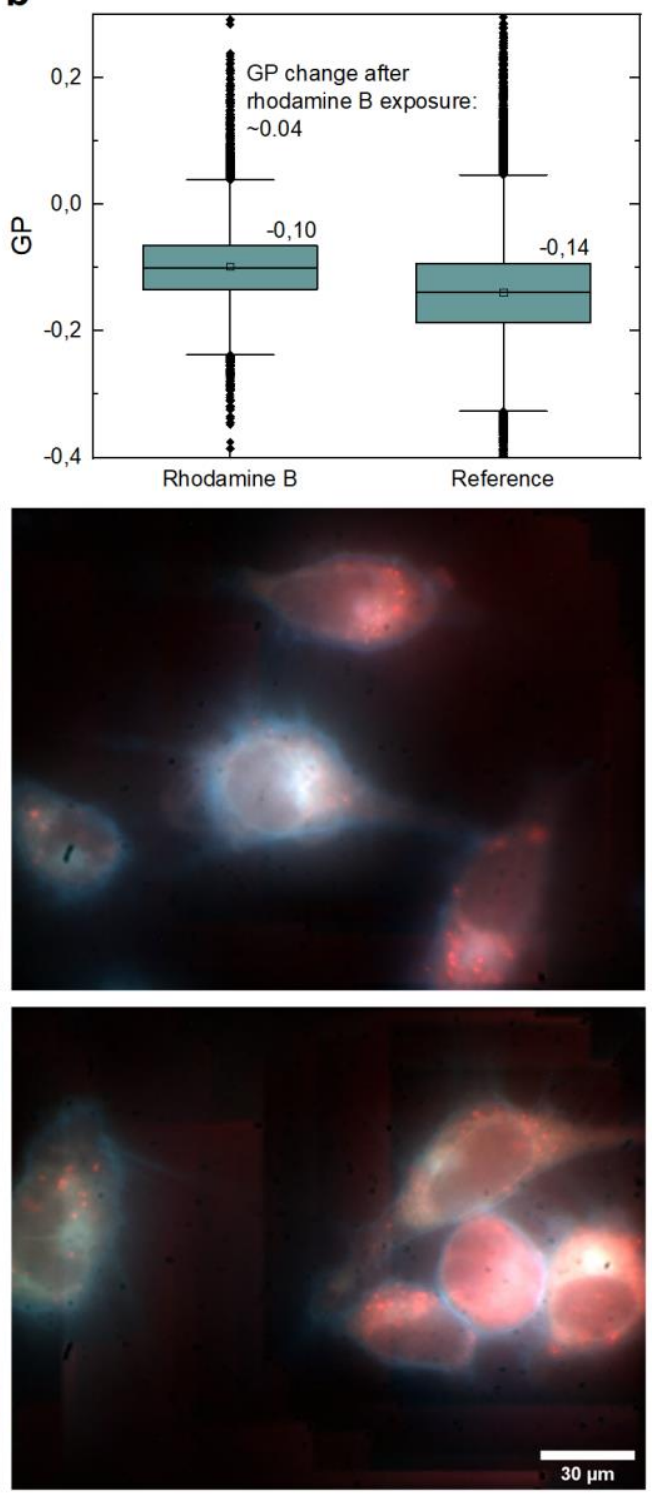

$30 \mu \mathrm{m}$

d
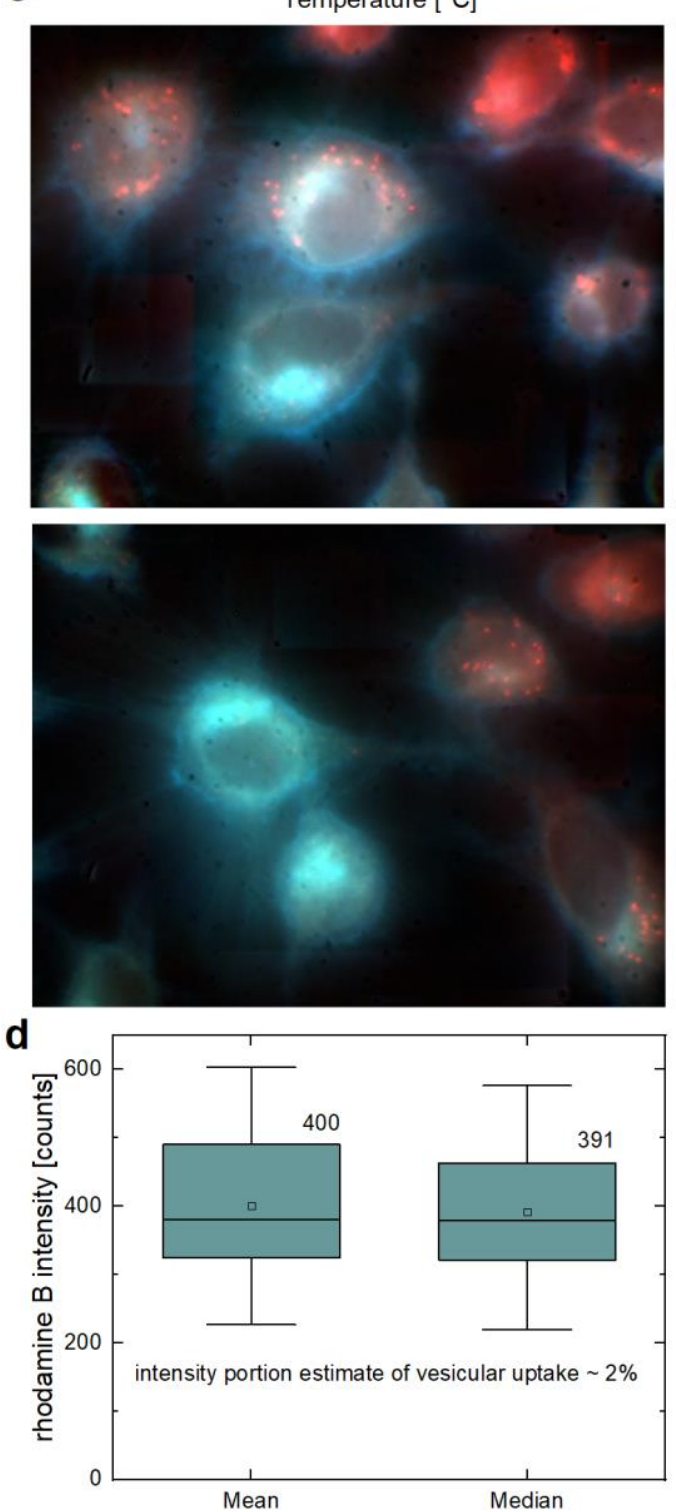

SI-Figure 1 Rhodamine B temperature dependence, GP dependence on rhodamine B and microscopy images of HeLa cells a Temperature dependence of Rhodamine $B$. The intensity values were normalized to $22^{\circ} \mathrm{C}$. The intensity of the $10^{\circ} \mathrm{C}$ uptake experiments were divided by 1.5 . The $37^{\circ} \mathrm{C}$ experiment was corrected for a factor of 0.6 b Mean GP value of cells stained only with Laurdan and rhodamine B exposed cells at $22^{\circ} \mathrm{C}$. Rhodamine $\mathrm{B}$ exposure increases cell membrane lipid order by 0.04. c 100x magnification fluorescence microscopy image of Laurdan stained HeLa cells after exposure to rhodamine B. Uniform rhodamine B intensity in the cytosol and rhodamine B loaded vesicular structures suggest two membrane transport pathways into the cell. $\mathbf{d}$ Median and mean rhodamine B intensity measured in high magnification micrographs. The median intensity is less sensitive to intensities arising from bright vesicular structures. 
a
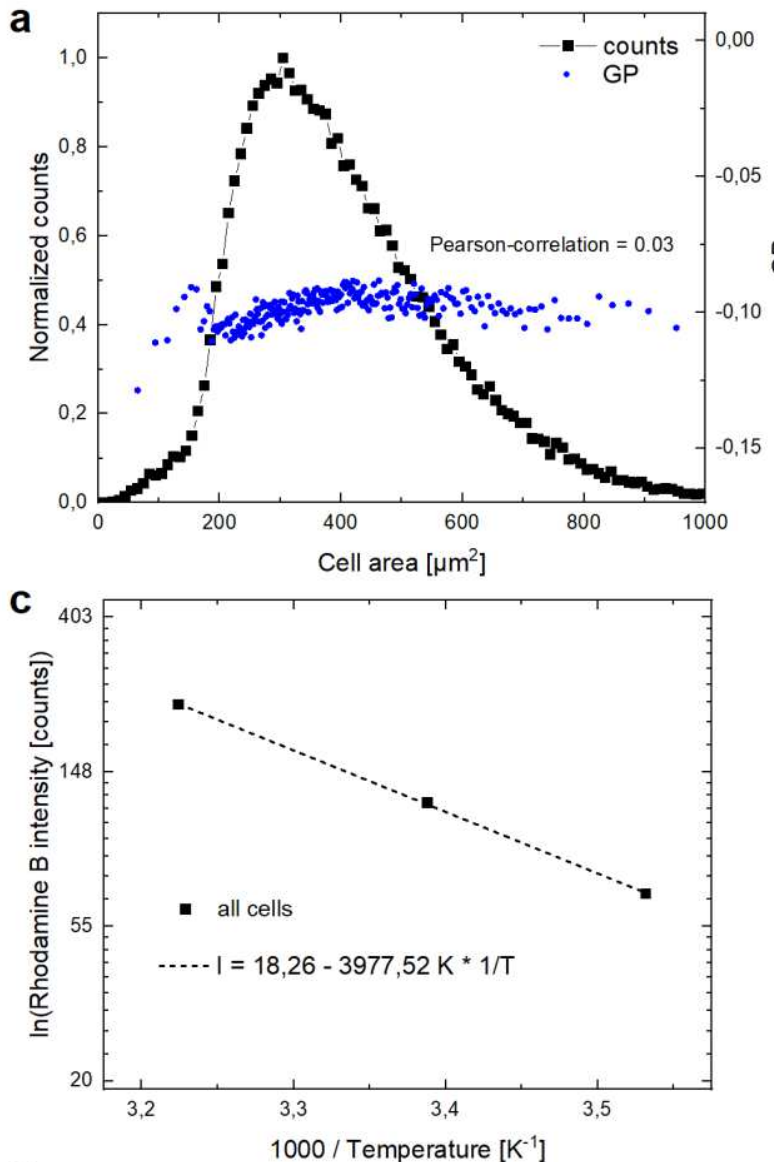

e

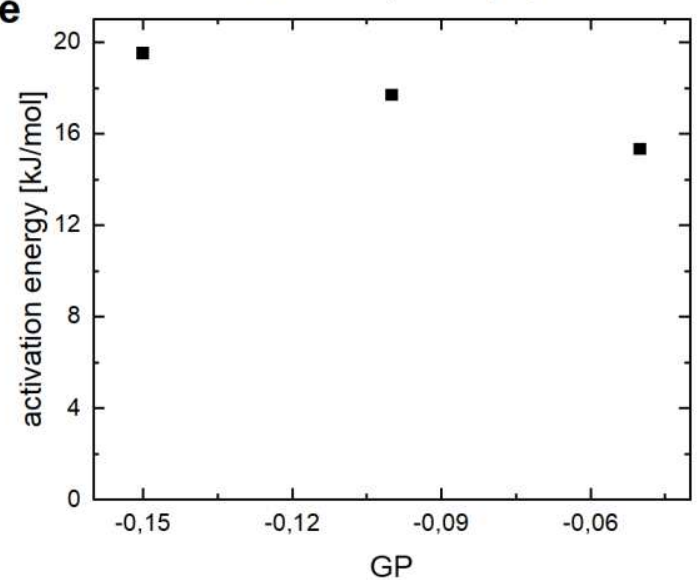

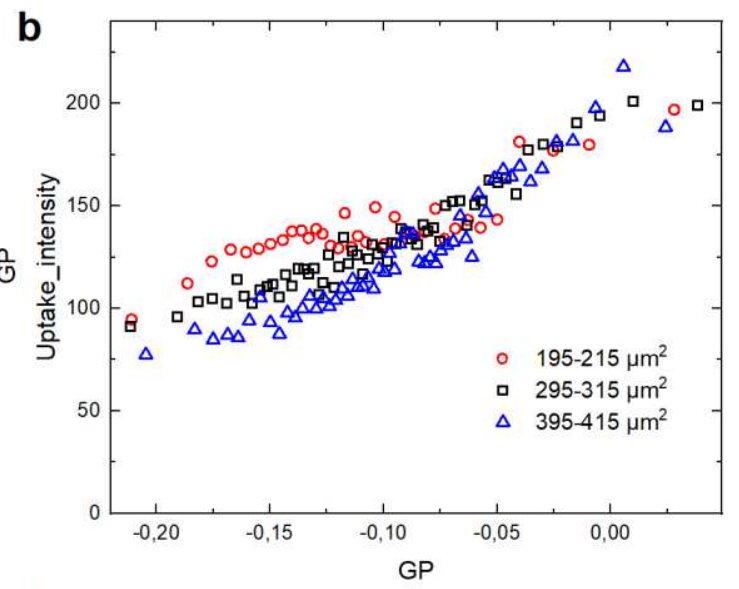

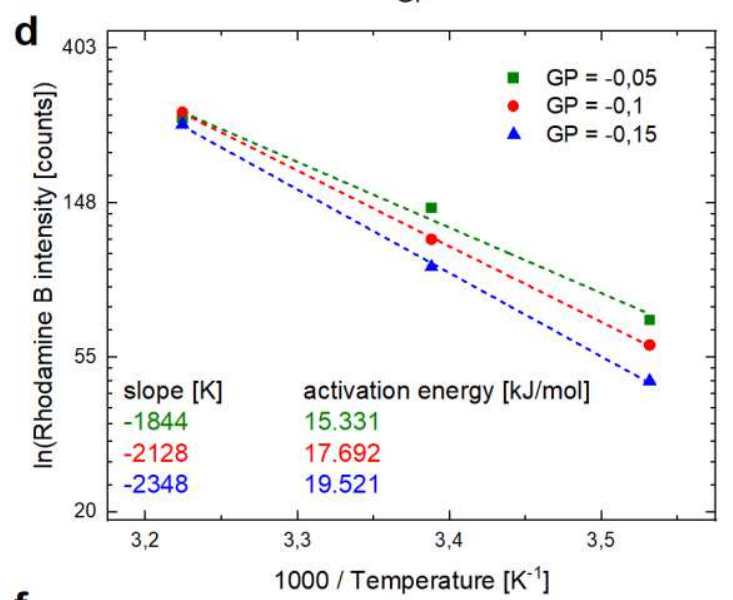

f

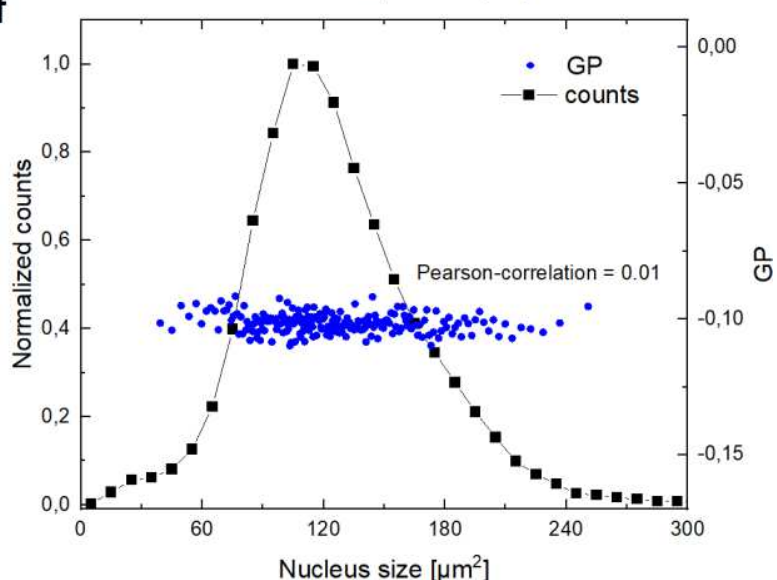

SI-Figure 2 GP correlation analysis of cell/nucleus size and Arrhenius plots of rhodamine B uptake experiments a Histogram of cell areas and GP dependency on cell area. A pearson-correlation coefficient of 0.03 indicates low correlation of the corresponding quantities. b Rhodamine B intensity as a function of GP for different cell area intervals. Independent on the cell size Rhodamine B uptake increases with increasing lipid chain order. c Arrhenius plot of the mean rhodamine B intensity of the uptake experiments conducted at 10,22 and $37^{\circ} \mathrm{C}$. The fit parameters are used in the Arrhenius law plotted in Fig. $3 \mathrm{e}$ d Arrhenius plot of the mean rhodamine B intensity data shown in c for different GP intervals. The slope of the according linear approximations can be converted to activation energies. e GP dependent activation energies obtained from the data shown in $\mathrm{d}$. With increasing lipid order the activation energy necessary to cross the cell membrane decreases. $\mathbf{F}$ Nucleus size distribution of the Hoechst 33342 uptake experiments and GP dependency on the nucleus size. There is no pronounced correlation of nucleus size and GP visible in agreement with a pearson-correlation coefficient of $r=0.01$. 

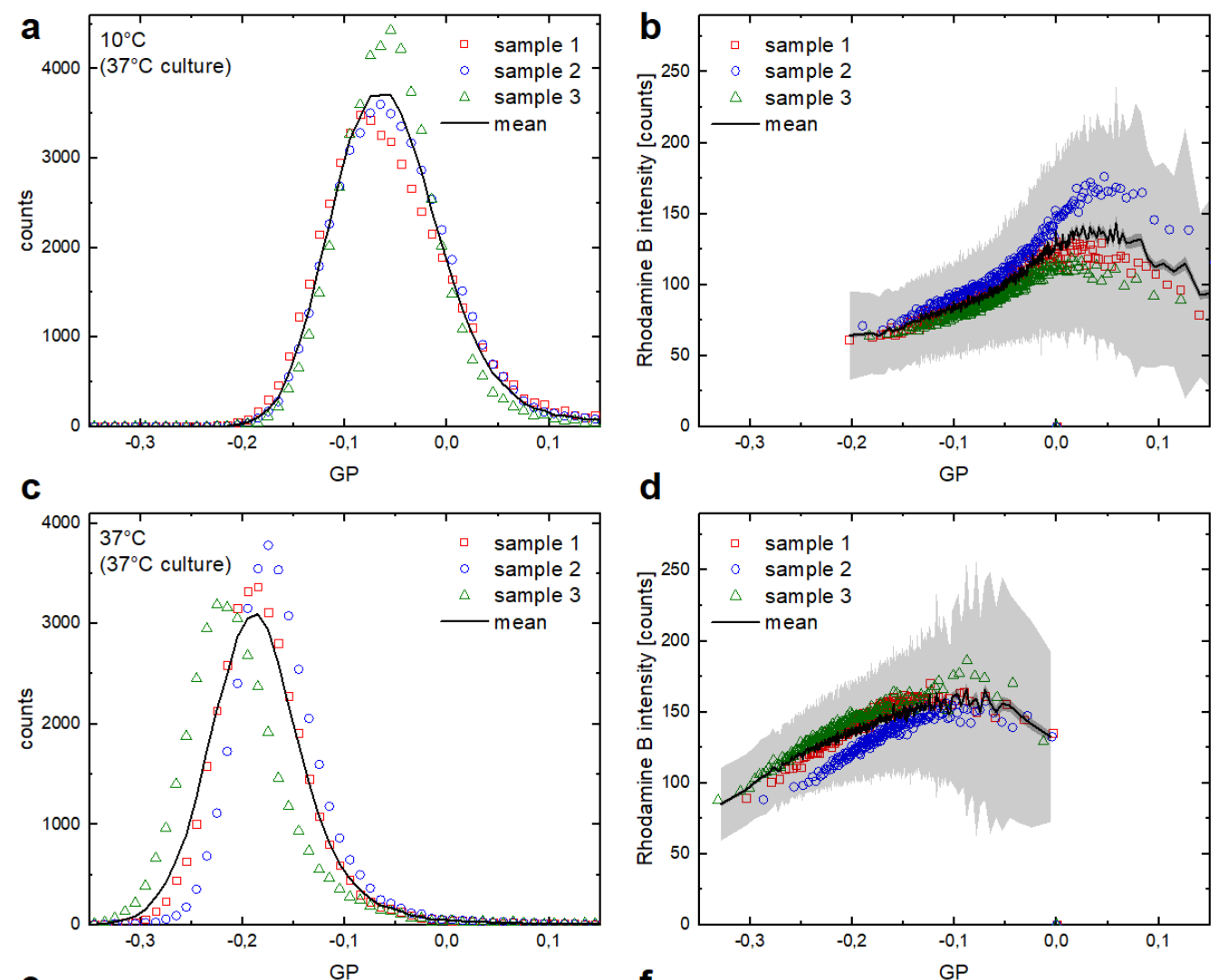

d
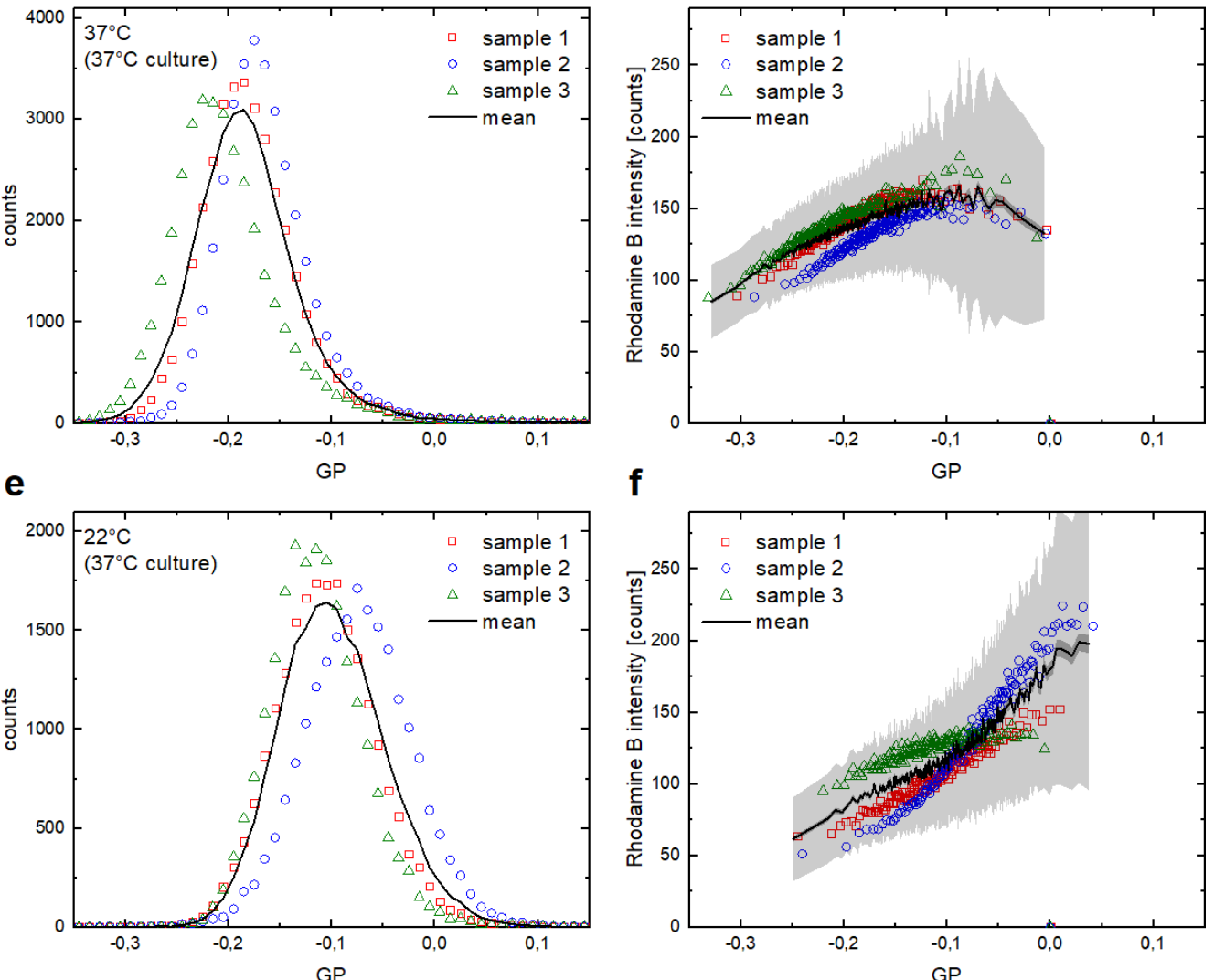

$\mathbf{f}$
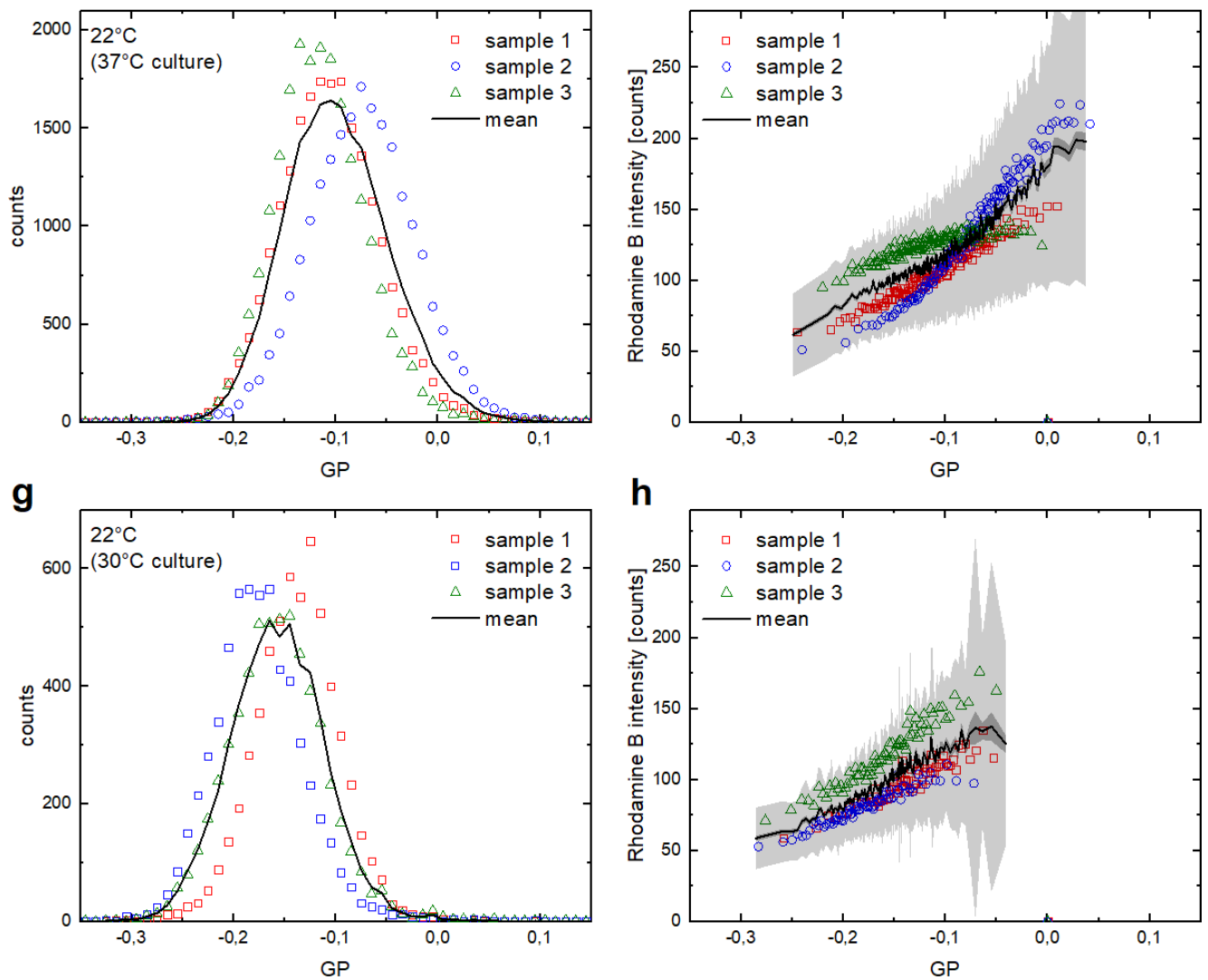

SI-Figure $3 \mathrm{GP}$ histograms with bin size 0.01 of rhodamine $\mathrm{B}$ uptake experiments conducted three times independently at $10^{\circ} \mathrm{C}\left(37^{\circ} \mathrm{C}\right.$-culture), $37^{\circ} \mathrm{C}\left(37^{\circ} \mathrm{C}\right.$-culture $), 22^{\circ} \mathrm{C}\left(37^{\circ} \mathrm{C}\right.$-culture) and $22^{\circ} \mathrm{C}\left(30^{\circ} \mathrm{C}\right.$-culture). Rhodamine B intensity data of the according experiments each reduced to the square root of the number of cells. Symbols indicate mean values, light gray colored bands standard deviation and dark gray colored bands standard error. a, $\mathbf{b}$ Number of cells in each sample: 50015. Each sample reduced to $50015^{0.5}=224$ datapoints (red, green and blue symbols). Total number of cells: $3 \times 50015=150045$. All experiments reduced to $150045^{0.5}=387$ datapoints (black line) $c$, $\mathbf{d}$ Number of cells in each sample: 34919. e, f Number of cells in each sample: 20986. g, h Number of cells in each sample: 5859. 